\title{
APURDUTI
}

Euskal ikerketen aldizkaria | Revue d'études basques |

Revista de estudios vascos | Basque studies review

$16 \mid 2012$

Numéro XVI

\section{La base de données NORANTZ : vers un observatoire des parlers basques d'Aquitaine}

Irantzu Epelde, Bernard Oyharçabal et Jasone Salaberria

\section{OpenEdition \\ Journals}

Édition électronique

URL : http://journals.openedition.org/lapurdum/2353

DOI : 10.4000/lapurdum.2353

ISSN : 1965-0655

Éditeur

IKER

Édition imprimée

Date de publication : 1 octobre 2012

Pagination : 27-67

ISSN : 1273-3830

Référence électronique

Irantzu Epelde, Bernard Oyharçabal et Jasone Salaberria, «La base de données NORANTZ : vers un observatoire des parlers basques d'Aquitaine », Lapurdum [En ligne], 16 | 2012, mis en ligne le 03

décembre 2014, consulté le 01 mai 2019. URL : http://journals.openedition.org/lapurdum/2353 ; DOI : 10.4000/lapurdum.2353 


\title{
La base de données
}

NORANTZ : vers un

observatoire des parlers

basques d'Aquitaine

\author{
Irantzu EPELDE ${ }^{1}$, Beñat OYHARÇABAL ${ }^{2}$ \\ $\&$ Jasone SALABERRIA ${ }^{3}$ \\ IKER (UMR 5478) $)^{1,2,3}$, CNRS $^{2}$
}

\section{Le contexte sociolinguistique ${ }^{1}$}

Jusqu'à une date récente, les recherches sur la variation dans le domaine du basque ont été réalisées principalement dans une perspective diatopique. Initiées il y un siècle et demi par L. Bonaparte, pionnier de ce type de travaux en Europe à cette époque, et auteur d'une célèbre carte des dialectes et sous-dialectes du basque (1869), les recherches réalisées dans le domaine de la variation depuis lors, en dehors des travaux sur l'histoire de la langue (Michelena 1976, 1988, Lakarra 2006, 2011, Igartua 2012 ...), ont presque exclusivement porté jusqu’ici sur les dialectes traditionnels ou des parlers locaux (Aurrekoetxea 1992, Ibarra 1995, Zuazo 1998, 2003, Camino 1997, 2004, Coyos 1999, Epelde 2004, Montoya 2004, Makazaga 2010, entre de multiples autres références) et c'est encore à ce type d'études que les grands projets réalisés dans ce secteur au cours des dernières années ont été consacrés, comme notamment l'atlas linguistique des parlers basques traditionnels (Aurrekoetxea \& Videgain 1993, Euskaltzaindia $(2008,2010,2012)$.

Pourtant la réalité sociolinguistique depuis quelques décennies a connu des changements considérables en Pays Basque, particulièrement dans sa partie aquitaine. D'abord, en raison des évolutions relatives au nombre de locuteurs et surtout à leur proportion au sein de la population, mais aussi parce que les conditions de transmission ont connu des modifications très importantes, notamment du fait de l'affaiblissement de la densité du maillage social formé par les locuteurs bascophones actifs dans la plupart des zones bascophones traditionnelles du côté nord de la frontière. Paradoxalement, dans la zone la plus urbanisée, largement

1.- Adresse: www.norantz.org. Le projet a été réalisé grâce à un financement du Conseil régional d'Aquitaine, du Conseil Général des Pyrénées-Atlantiques et de l'Office Public de la Langue basque. 
située hors de la géographie des parlers historiques, et où la pratique du basque a pu se développer récemment dans des conditions nouvelles, des circonstances quelque peu analogues se retrouvent.

Par ailleurs, depuis environ quatre décennies se développe le basque standard, qui représente aujourd'hui pour de nombreux locuteurs, une référence importante. Parallèlement, l'influence des parlers ibériques, en particulier guipuscoans, les plus dynamiques à l'époque moderne, notamment au cours des dernières décennies, a été de plus en plus prégnante, en raison de la place importante qu'ont les locuteurs de ces parlers dans les médias audio-visuels et la littérature de plus forte diffusion, ainsi que dans le développement du basque standard. En Aquitaine, cette influence est renforcée par leur présence significative dans les instances d'enseignement et de transmission, présence elle-même encouragée par des phénomènes de migration, très naturels dans une zone frontalière. Ces phénomènes correspondent à des tendances fortes, et tout donne à penser que leurs effets se feront également sentir dans le futur.

Sans entrer dans le débat sur la nature profonde des relations entre contact des langues et changements linguistiques, il existe un large consensus pour considérer que le contact des langues est un facteur décisif des changements linguistiques. Il est également admis que les effets du contact des langues et des processus porteurs des modifications linguistiques croissent lorsque les espaces et circonstances d'utilisation d'une langue se dispersent et se réduisent. Dans le cas du Pays Basque aquitain (désormais PBA), on peut s'attendre a deux types d'incidence : l'une résultant du contact avec le français, l'autre consécutif au contact avec les parlers basques sociologiquement dominants.

Ainsi, le fait que tous les locuteurs bascophones d'Aquitaine soient bilingues et vivent dans leur immense majorité dans un environnement social principalement francophone laisse prévoir une incidence significative sur la langue socialement minorée dont ces locuteurs sont porteurs. Par ailleurs, de manière parallèle, l'influence des modèles linguistiques basques de plus grande diffusion ou prestige, bénéficiant dans d'autres parties du Pays Basque de la protection de la loi, se fait plus forte chez ces locuteurs de parlers en voie d'affaiblissement. Il y a donc, à côté des phénomènes de contact pouvant favoriser des changements de divergence par influence du français, d'autres facteurs favorisant eux des modifications de convergence chez les locuteurs bascophones.

Le tableau suivant rappelle quels étaient les résultats les plus significatifs de l'enquête sociolinguistique de 2011 en Iparralde. ${ }^{2}$ Ils montrent en particulier que dans la population de

2.- Vème enquête sociolinguistique. Communauté autonome d'Euskadi, Navarre et PBA. Vice-Ministère de la politique linguistique de la Communauté autonome et Office Public de la Langue basque. Saint-Sébastien, 2012, p. 10. 
16 ans et plus résidant en Pays Basque, ${ }^{3}$ et considérée dans sa globalité, le basque est une langue minoritaire, dans des proportions d'autant plus marquées que l'on approche des couches d'âges les plus jeunes: 30\% chez les plus de 65 ans, 14\% chez les $25-34$ ans. ${ }^{4}$

\begin{tabular}{|l|l|l|l|l|l|l|}
\hline Population $\geq 16$ & Total & $\geq 65$ & $50-64$ & $35-49$ & $25-34$ & $16-24$ \\
\hline Nombre & 238360 & 62266 & 55710 & 61365 & 32056 & 26964 \\
\hline Bilingues actifs & $21,4 \%$ & $30,6 \%$ & $24,4 \%$ & $05,1 \%$ & $13,9 \%$ & $17,6 \%$ \\
\hline Bilingues réceptifs & $09,1 \%$ & $07,6 \%$ & $07,2 \%$ & $09,8 \%$ & $12,3 \%$ & $11,3 \%$ \\
\hline Non-bascophones & $69,4 \%$ & $61,8 \%$ & $68,5 \%$ & $75,1 \%$ & $73,7 \%$ & $71,1 \%$ \\
\hline
\end{tabular}

Sur l'ensemble du territoire objet de l'enquête, ${ }^{5}$ la proportion des bascophones varie selon les zones géographiques. Les enquêtes sociolinguistiques réalisées distinguent trois zones:

- une zone dite BAB (Bayonne-Anglet-Biarritz), entièrement urbanisée, représentant la principale concentration urbaine de la région (près de 100000 personnes pour la population d'enquête: 98899 h.)

- une zone dite ici Labourd mixte, incluant la partie du Labourd non incluse dans le $\mathrm{BAB}$, zone qui contient, d'une part, des villes moyennes côtières (Saint-Jean-de-Luz, Hendaye), et, d'autre part, une zone rurale ou en cours de rurbarnisation; la population de cette zone dépasse les 100000 habitants (106 879 h.)

- la zone constituée par les provinces de Basse-Navarre et Soule (désormais BN \& S), peu urbanisée, et moins peuplée (32 582 h.).

3.- La population est celle habitant le Pays Basque et âgée de 16 ans et plus. Elle inclut les personnes non-natives mais résidant en Pays Basque au moment de l'enquête. Ce nombre n'est pas évalué pour cette enquête, mais il est considéré comme relativement important, le Pays Basque étant de plus en plus une zone d'accueil pour de nombreuses personnes adultes non originaires. Il va sans dire que la grande majorité de ces populations, non bascophones par définition lors de leur venue, ne le devient pas, puisque la situation sociolinguistique ne permet une acculturation linguistique naturelle des non-natifs, contrairement à ce qu'il en était jusqu'au milieu du XXème siècle, du moins en zone rurale, pour les arrivants jeunes.

4.- On remarquera dans le tableau que la proportion de bascophones dans la génération suivante des 16-14 ans est en légère progression (17\%). C'est le résultat des efforts réalisés dans le domaine de l'enseignement du basque dans les années 1985-1995.

5.- Le territoire concerné est celui retenu pour délimiter l'entité administrative dite 'pays Pays-Basque'. Celle-ci englobe le territoire des trois provinces historiques, la ville de Bayonne, et quelques communes voisines de la périphérie bayonnaise sur la rive droite de l'Adour. 
La distribution des bascophones dans ces trois zones montre que c'est uniquement dans cette dernière zone que les bascophones ne sont pas en nette minorité, les locuteurs bascophones actifs représentant $52 \%$ de la population, contre $9 \%$ dans le BAB et $24 \%$ dans la zone mixte du Labourd. Il va sans dire que statistiquement tous les locuteurs bascophones sont également francophones.

\begin{tabular}{|l|c|c|c|c|}
\hline Zones & PBA & BAB & $\begin{array}{c}\text { Labourd } \\
\text { mixte }\end{array}$ & BN \& S \\
\hline Locuteurs & 238360 & 98899 & 106879 & 32582 \\
\hline Bilingues actifs & 51100 & 8561 & 25571 & 16968 \\
\hline Bilingues réceptifs & $22 \%$ & $09 \%$ & $24 \%$ & $52 \%$ \\
\hline Non-bascophones & 21742 & 5929 & 11231 & 4582 \\
\hline & $09 \%$ & $06 \%$ & $11 \%$ & $14 \%$ \\
\hline
\end{tabular}

5ème enquête sociolinguistique (2011)

Cette influence du facteur géographique apparaît également, bien que de manière moins brutale, lorsque l'on considère la distribution des bascophones dans les trois zones. En effet, les bascophones - du moins les actifs- habitent pour moitié dans la zone dite intermédiaire du point de vue de l'urbanisation et appelée ici Labourd mixte, un tiers dans la zone la plus rurale (Basse-Navarre et Soule), et un sixième dans la zone de plus forte concentration urbaine, ainsi que le montre le tableau qui indique comment se répartissent les bascophones selon leur zone d'habitation:

\begin{tabular}{|l|c|c|c|}
\hline Zones & BAB & Labourd mixte & Basse-Navarre \& S \\
\hline Bilingues actifs & $16,7 \%$ & $50,1 \%$ & $33,2 \%$ \\
\hline Bilingues réceptifs & $27,2 \%$ & $51,6 \%$ & $21,1 \%$ \\
\hline
\end{tabular}

Ces éléments lorsqu'ils sont placés dans une perspective dynamique laissent apparaître une dégradation régulière de la situation sociolinguistique du basque, même si les efforts réalisés au cours des dernières années commencent à produire quelques effets significatifs. Les résultats corrigés de l'enquête de 1991 évaluaient le nombre des bascophones actifs dans 
la population de 16 ans et plus à 59 900. ${ }^{6}$ Dix-neuf ans plus tard les bascophones actifs sont évalués à $51100 .{ }^{7}$ Selon ces évaluations, le nombre des bascophones a donc baissé de près de $15 \%$ en un peu moins d'une vingtaine d'années.

Sur une échelle correspondant au siècle, l'affaiblissement actuel est la poursuite d'un processus dans lequel les générations nées après les deux guerres mondiales ont joué un rôle décisif, car c'est chez elles que la rupture de la transmission a été la plus sensible. Le résultat est la fréquence avec laquelle se rencontrent des familles où le basque n'est connu que par les générations les plus âgées, avec, en conséquence, un nombre également significatif de locuteurs bascophones de première langue qui, dans la plupart des usages, n'ont pas recours au basque ; peu à peu, celui-ci devient pour eux une langue inusitée, dans laquelle leur compétence est amoindrie selon un processus bien décrit dans les situations d'attrition linguistique résultant d'un language shift. De façon complémentaire, il n'est pas rare aujourd'hui qu'en raison des efforts réalisés pour l'apprentissage scolaire du basque, certains locuteurs n'utilisent guère leur compétence en basque qu'avec les enfants, ou dans des contextes très réduits, plus ou moins ritualisés.

Bien que le projet Norantz ne concerne pas la sociolinguistique quantitative, et ne porte pas non plus sur l'étude des choix de langues par les locuteurs bilingues, le rappel un peu lourd de ce contexte était nécessaire pour comprendre le contexte dans lequel le projet Norantz a été réalisé.

\section{Description du PROJET.}

L'objectif du projet NoRANTZ, réalisé entre 2009 et 2011 au centre IKER, ${ }^{8}$ a été d'offrir à travers une base de données, une image générale et synthétique de la grammaire des locu-

6.- Les résultats publiés de l'enquête de 1991 (la première réalisée à l'époque moderne) faisaient apparaitre un nombre de bascophones plus importants (69 100). La mise en perspective de ces résultats à partir des enquêtes réalisées par la suite en tenant compte de la distribution par groupes d'âge a conduit les responsables des enquêtes à publier des résultats corrigés (que nous retenons ici). 'Réflexions sur le PBA', 5 eme enquête sociolinguistique, 2011. Le Gouvernement basque et l’Office public de la Langue basque. Télédéchargeable à l'adresse suivante:

<http://www.mintzaira.fr/fileadmin/documents/Aktualitateak/096_PEN_REFLEXIONS_3.pdf>

7.- La première évaluation chiffrée du nombre de bascophones dans le département date de 1808. Elle fut publiée en 1809 dans l'Annuaire présenté à S. M. l'Empereur et Roi par le Bureau des Longitudes, année 1808 (Oyharçabal 1993). L'évaluation du nombre des bascophones effectué par l'administration préfectorale, s'élevait à 108 000, soit plus du double du dénombrement effectué 200 ans plus tard. Dans la seconde moitié du XIXème siècle les spécialistes proposaient des évaluations en nette augmentation. F. Michel en 1857 proposait une estimation de 140 000, et E. Reclus en 1867 de 120 000. Velasco (1879) avançait une évaluation nettement plus basse (80 000), mais elle est certainement bien moins fiable que celles de Michel, très bon connaisseur du PBA, et d'E. Reclus, excellent géographe qui s'était intéressé à la langue basque. Lévaluation proposée par Yrizar (1973) pour la fin des années 1960 marquait dèjà un net affaiblissement : 77.848 , tendance qui s'est poursuivie jusqu'à aujourd'hui.

8.- Le projet Norantz a bénéficié d'un financement de la Région Aquitaine, du département des PyrénéesAtlantiques et de l'Office public de la Langue basque. 
teurs bascophones du PBA, c'est-à-dire bilingues français-basques, en tenant compte de la diversité des types de locuteurs, et selon une méthodologie qui devait permettre sa répétition dans le futur, de façon à suivre les changements opérés ou en cours, et donc de créer les bases d'un observatoire de l'évolution de la grammaire des parlers basques de France.

Les études linguistiques synthétiques supposent en principe la réalisation d'un nombre suffisant d'études ponctuelles à partir desquelles il est possible de synthétiser les éléments recueillis. Cette façon de procéder, dans le cas des territoires concernés, conduisait toutefois en pratique à une impasse, en raison du peu d'études ponctuelles du type d'Epelde (2004) réalisées au cours disons des dix ou vingt dernières années, et le peu de possibilité de voir leur nombre augmenter dans les années futures.

Pour obtenir une représentation, minimalement fiable, de la compétence grammaticale des locuteurs du PBA, dans leur diversité, à la fois géographique, dialectale et générationnelle, l'idée a donc été de demander à un groupe significatif de locuteurs (près d'une soixantaine), sélectionnés à partir de critères d'âge et de lieux de vie principalement, de répondre à un questionnaire couvrant un large éventail de la morphosyntaxe du basque, et un aspect de sa phonologie. Par ailleurs, pour s'assurer de la faisabilité du projet sur une période relativement brève (36 mois au total) et des moyens humains, certes significatifs, mais également limités (deux chercheurs postdocs à temps plein en charge du recueil des données et de l'alimentation de la base de données), le recours à la traduction, malgré ses limites et les difficultés qu'il engendre, a été adopté. Ce mode de collecte des données permettra par ailleurs de comparer les données recueillies sur une période étendue à l'avenir.

\subsection{La typologie des locuteurs.}

Lidée de base du projet étant de pouvoir repérer les tendances majeures de l'évolution des parlers de façon la plus générale possible, une typologie des locuteurs bascophones adultes du PBA a été dressée à partir de deux types de facteurs relativement simples à contrôler : l'âge et le lieu d'habitation. ${ }^{9}$ Pour le groupe concerné par ce point, un troisième critère a été également pris en compte : celui du type de scolarité suivi relativement à l'enseignement du basque : immersion sur toute la scolarité obligatoire, ou non.

\section{Le critère de l'âge}

Relativement à l'âge, trois groupes ont été constitués avec, comme repères de rupture, la fin de la guerre mondiale, et l'après 1968, considérés comme des périodes marquant d’importants changements dans la vie sociale:

- le groupe des personnes nées avant la fin de la guerre, est caractérisé dans la base

9.- Pour élaborer la typologie des locuteurs, les auteurs ont notamment bénéficié de la collaboration de B. Haddican. 
de données comme celui 65 ans et plus, et il est constitué de 15 locuteurs. Il rassemble deux sous-groupes de 6 personnes nées entre les deux guerres, respectivement avant 1930 et 1940, et ayant effectivement 70 ans au minimum en 2009, et trois personnes entre 1941 et 1944 (durant la guerre). Le spectre des naissances couvre en gros deux décennies environ (entre 1925 et 1944).

- Le groupe des locuteurs d'âge intermédiaire comprend 12 locuteurs nés après-guerre (11 après 1950 et 1 en 1946) et avant 1971, avec en noyau les personnes nées durant les années 50 (8 locuteurs).

- Le groupe des locuteurs jeunes comprend 32 informateurs nés à une exception près après 1979, et donc ayant 30 ans ou moins en 2009 (à l'exception d'un informateur né 1976 et rattaché à ce groupe). Linformateur le plus jeune, né en 1993, avait 17 ans lors de la réalisation de l'enquête, cinq informateurs au total ayant moins de 20 ans en 2009.

Comme on le voit le groupe le plus important (un peu plus de la moitié des informateurs) est celui des 30 ans et moins, ce qui s'explique, d'une part, par l'orientation de la base de données vers l'identification de la dynamique des changements linguistiques, portés plus spécialement par les jeunes générations; d’autre part, par la plus grande casuistique sociolinguistique résultant de la prise en compte du mode de scolarisation relativement au basque (système d'immersion ou non), et qui ne pouvait être pertinent dans les deux autres groupes, le basque étant totalement exclu du système scolaire à l'époque ou ces générations ont été scolarisées. En effet, le groupe des jeunes distingue systématiquement ceux ayant bénéficié d'un enseignement du basque par immersion : 18 informateurs, et ceux pour qui cela n'a pas été le cas : 14 informateurs (voir infra pour l'explication de cet écart).

Afin de mieux identifier chacun de ces groupes, et leurs tendances caractéristiques, on s'est attaché, dans la mesure du possible, à marquer les écarts entre chacun des groupes, comme il est d'usage dans ce type d'enquête (au moins cinq ans, à une exception près). Par ailleurs, pour chaque groupe d'âge, on a veillé à une certaine répartition longitudinale. De façon plus informelle, on s'est efforcé lors de la sélection des informateurs de varier les expériences relativement à la langue : inclusion de personnes ayant fait de long séjours à l'étranger et ayant récupéré le basque ensuite, ou au contraire ayant toujours eu une vie familiale en basque; personnes ayant appris le basque à l'âge adulte, ou ne l'ayant appris qu'à l'école, ou au contraire par la seule transmission familiale ; etc. Cet effort regardant la variété a porté également sur le conditionnement dialectal (au sens traditionnel du terme). Ainsi qu'indiqué plus haut, le projet Norantz ne concerne pas la dialectologie traditionnelle, en voie de grande transformation (sans que certaines spécificités ne disparaissent pour autant, comme dans le cas du souletin). Les éléments dialectaux en eux-mêmes n’ont donc pas été inclus dans les critères définissant la typologie des locuteurs. Cependant, par la réalisation des enquêtes sur la totalité du territoire bascophone (voir carte infra), il a été possible, d'inclure des informateurs porteurs des principales variétés parlées, et également aussi ceux porteurs de variétés mélangées, en raison des nouvelles conditions de vie (déplacement des personnes, mariages mixtes, scolarisation en basque standard, etc...). Les profils dialectaux des locuteurs sont indiqués 
dans le profil associé à chaque informateur dans la base de données.

Eu égard à la nature des données que l'on voulait recueillir, aucune sélection quant à la qualité des informateurs du point de vue de la maîtrise de la langue n'a été effectuée. Les personnes remplissant les critères sociologiques indiqués, acceptant et en mesure de répondre au questionnaire ont été retenues. La longueur du questionnaire, sa variété, sa difficulté parfois, compte tenu de ce qu'il fallait traduire, sur l'instant et devant magnétophone, les nombreuses phrases qui étaient soumises par l'enquêteur, font que, de facto, seuls des locuteurs ayant une compétence minimale ont pu participer à l'enquête. Mais, ni la 'correction' des réponses, ni la compétence des informateurs en matière de communication ou sur le plan lexical, n'ont été des facteurs de sélection. En aucune façon, des personnes sachant 'bien' le basque ou des locuteurs idéaux ayant une maîtrise remarquable du basque, n’ont été recherchées. Certaines personnes retenues peuvent évidemment s'approcher d'un tel profil, mais cela n'a pas été la raison de leur sélection. Au demeurant, d'autres informateurs ont une maîtrise limite de la morphosyntaxe de la langue, comme on peut le constater dans les réponses de certaines informateurs, ayant eu une expérience linguistique peu favorable à l'usage du basque. C'est évidemment le résultat de la variété des parcours individuels (relativement à la pratique de l'euskara), et il nous a paru utile que la base de données recueille ce type de données. En effet, qui connait le monde de la bascophonie aujourd'hui, sait qu'il est aussi constitué de façon non marginale de locuteurs ayant eu des expériences linguistiques très irrégulières. La base de données devant recueillir les différents types de parlers, ces locuteurs n'ont pas été exclus de la base de données. A l'inverse, en raison de ce même souci de recueillir des témoignages des locuteurs ordinaires, les professionnels de la langue basque (comme les enseignants, les techniciens-traducteurs ou les journalistes) ont été écartés.

\section{Le critère géographique.}

Relativement au lieu d'habitation, deux grandes zones ont été distinguées : celle de grande urbanisation (agglomération urbaine Bayonne-Biarritz-Anglet, désormais BAB) et une zone incluant villes petites et moyennes et campagne. Cette seconde zone, au départ, distinguait le monde rural proprement dit, mais, en cours d'enquête, la difficulté de classement, résultant en bonne partie des phénomènes de rurbanisation en pleine extension vers l'intérieur du Pays Basque, a conduit à simplement ne retenir que l'opposition entre le BAB et le reste du territoire. La carte indiquant les lieux d'habitation des informateurs reflètent la répartition sur l'ensemble du territoire: 


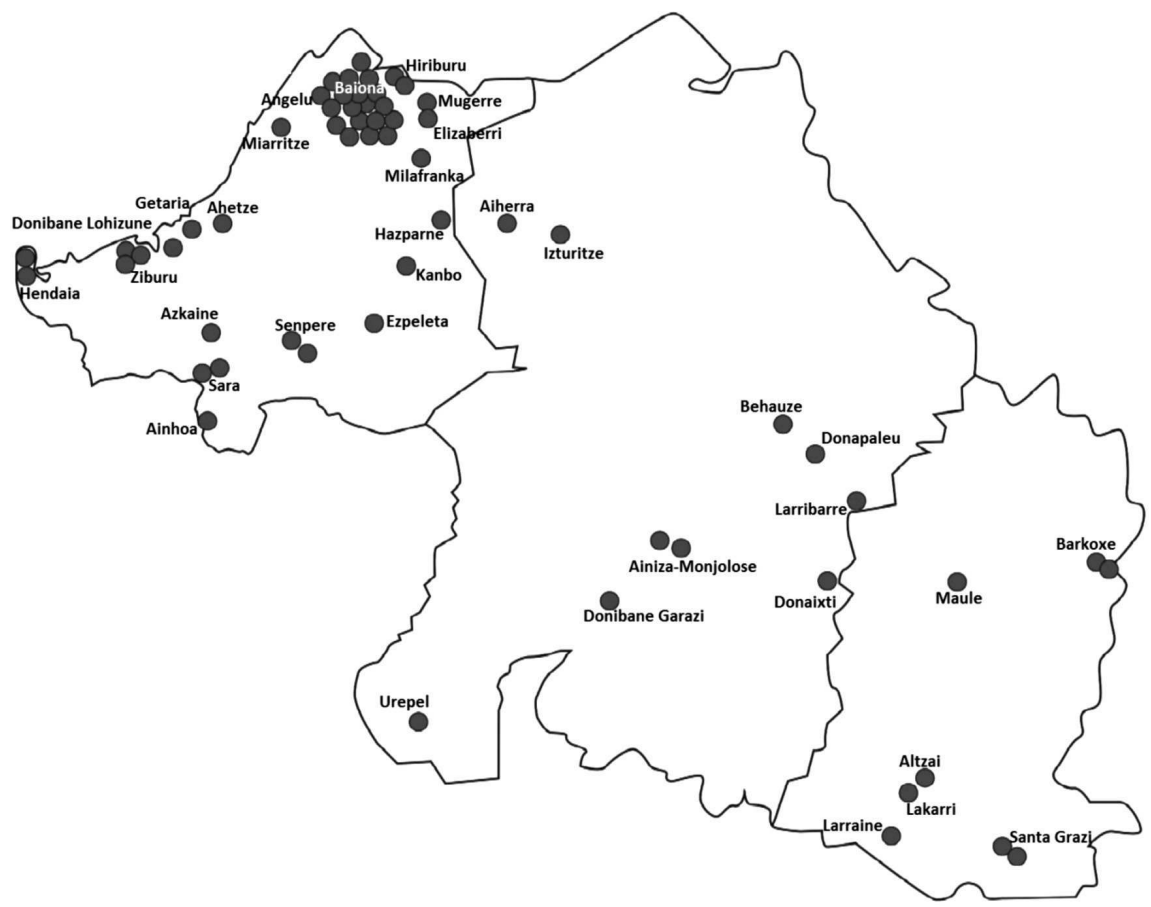

Carte des lieux de résidence des informateurs

Le croisement du critère géographique (2 zones) avec celui de l'âge (3 groupes) créait une typologie distinguant six groupes de locuteurs. Cependant, comme indiqué plus haut, pour le groupe des moins de 30 ans, le mode de scolarisation a été également pris en compte, d'où, au final, huit groupes. Pour ces huit groupes, l'idée était d'obtenir un nombre d'informateurs variant entre 5 et 8 pour les deux groupes les plus âgés et autour de la dizaine pour les plus jeunes (groupe d'âge le plus à même de marquer des éléments d'évolution). Cet objectif a pu être atteint, sauf pour le groupe des jeunes informateurs habitant en agglomération (total de 8), et notamment celui n'ayant pas suivi une scolarisation par immersion (2 informateurs). ${ }^{10}$ Cet état de chose correspond très certainement à une réalité : la difficulté de rencontrer des locuteurs actifs (acceptant et en mesure de répondre à un long questionnaire

10.- Lun de ces deux informateurs a appris le basque par les cours d'apprentissage associatif. Le second, né dans une bourgade de l'intérieur en Basse-Navarre, mais élevé dans l'agglomération BAB, a acquis dans le cadre de la transmission familiale. 
comme celui de l'enquête), qui soient nées après 1979, aient vécu dans la zone BAB, et n'aient pas suivi une scolarité par immersion. En un mot, ceci reflète la difficulté de la transmission du basque dans le seul cadre familial (c'est-à-dire sans l'appui scolaire), en dehors des zones rurales ou proches du monde rural. Il est d'ailleurs probable que ce phénomène est en cours de généralisation, y compris dans ces derniers territoires également.

Le tableau ci-dessous indique donc la typologie des locuteurs dont les réponses sont recueillies dans la base de données Norantz.

\begin{tabular}{|c|c|c|c|c|}
\hline \multicolumn{4}{|c|}{ Types de locuteurs } \\
\hline Âge & \multicolumn{2}{|c|}{ Agglomération } & \multicolumn{2}{c|}{ Petites villes et campagne } \\
\hline scolarisation & + immersion & - immersion & + immersion & - immersion \\
\hline+65 & - & 7 & - & 8 \\
\hline $40-60$ & - & 5 & - & 7 \\
\hline-30 & 6 & 2 & 11 & 13 \\
\hline
\end{tabular}

Typologie des locuteurs informateurs de la base de données NoranTz

Chaque informateur est identifié par un nom d'anonymation. Les principales caractéristiques sociolinguistiques associées à ces noms sont immédiatement visibles pour l'utilisateur de la base de données. En voici trois exemples:

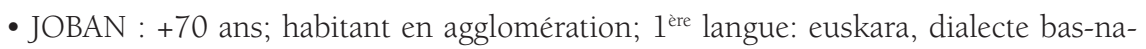
varrais; scolarité en français.

- PEOLO : -30 ans; a habité une petite ville hors du Pays Basque avec des séjours réguliers en milieu rural bascophone; $1^{\text {ère }}$ langue: français; basque appris avec les grands-parents; scolarité en français.

- KABA : -30 ans; habitant en agglomération; $1^{\text {èr }}$ langue: euskara, dialecte bas-navarrais; scolarité bilingue par immersion.

\subsection{La méthode d'élicitation des données et le questionnaire}

La méthode d'élicitation des données est largement déterminée par l'idée de base du projet : à savoir la constitution d'une base de données permettant de repérer les changements se produisant dans les divers domaines de la morphosyntaxe et, secondairement, la phonologie du basque, à travers les générations de locuteurs actuels. Il fallait pour cela un questionnaire très structuré permettant de recueillir des réponses de manière semblable chez plusieurs dizaines de locuteurs, de façon à pouvoir les comparer, éventuellement lors d'enquêtes réalisées à des époques différentes. Afin de répondre à cet objectif, le questionnaire NoranTz a été entièrement basé sur la traduction de phrases ciblant chacune une forme morphologique ou phonologique ou une structure particulière.

La traduction correspond à un choix méthodologique largement utilisé pour étudier 
la variation au sein d'une langue chez les locuteurs bilingues tels que ceux composant la population bascophone actuelle. Au demeurant l'élicitation par traduction a une longue tradition dans les études basques, en particulier pour les études comparatives internes au basque, comme le montrent les travaux de Bonaparte et les recueils de Bourciez et Sacaze, au XIXème siècle. Dans le contexte des études basques, l'originalité de la méthodologie utilisée pour alimenter la base de données Norantz résulte surtout de ce qu'elle a porté principalement sur la morphosyntaxe, et de ce qu'elle a été basée sur des traductions orales permettant de créer des documents sonores, directement accessibles aux utilisateurs de la base de données.

Le questionnaire couvre, d'une part, la morphosyntaxe, et, d'autre part, les éléments de la phonologie les plus susceptibles d'être affectés par les phénomènes de contact des langues. Même si la faisabilité du projet obligeait à une réduction des questions à un nombre raisonnable, compte tenu des moyens, les questions portant sur la morphosyntaxe couvrent un très large éventail, ce qui implique inévitablement une relative superficialité. Le but, en effet, n'est pas de réaliser une analyse pour chacune des structures, mais de permettre de repérer les changements en cours, pour l'analyse desquels il faudra bien sûr réaliser des travaux supplémentaires d'une autre nature. ${ }^{11}$

La structure du questionnaire morphosyntaxique distingue trois grands chapitres : ${ }^{12}$

- la morphologie flexionnelle des syntagmes nominaux (81 questions),

- la morphologie flexionnelle verbale (159 questions),

- la syntaxe (323 questions).

Si on y ajoute 5 questions relatives à la morphonologie, cela fait un total de 568 questions. Voici, le descriptif général du questionnaire :

\section{A. Morphologie nominale}

A.1. Désinences casuelles et post-positionnelles : 74 questions

A.2. Démonstratifs : 7 questions

11.- Un projet de questionnaire ayant été élaboré par les responsables du projet au centre IKER (voir note 11), il fut présenté à un séminaire de travail rassemblant 25 linguistes basques, en particulier de l'Université du Pays Basque et de l'Université publique de Navarre (Bayonne, 2009/05/15). Le questionnaire définitif a été arrêté à la suite des observations, nombreuses et très constructives, faites par les assistants, à l'occasion de cette journée.

12.- Le questionnaire morphosyntaxique a spécialement bénéficié de la collaboration des linguistes du centre IKER associés au projet: et U. Etxeberria, R. Etxepare et X. Videgain. 


\section{B. Morphologie verbale}

B.1. Sans arguments personnels : 56 questions

B.2. Entre arguments personnels : 32 questions

B.3. Potentiel : 29 questions

B.4. Suppositives et conditionnelles hypothétiques : 18 questions

B.5. Formes verbales synthétiques : 5 questions

B.6. Marques aspectuelles : 8 questions

B.7. Formes allocutives : 11 questions

\section{Syntaxe}

C.1. Omission / réalisation des formes pronominales : 54 questions

C.2. Emploi du déterminant selon les contextes syntaxiques : 17 questions

C.3. Lexpression du haut-degré : 7 questions

C.4. Accord numéral des quantifieurs avec le verbe : 20 questions

C.5. Distributifs : 4 questions

C.6. Le marquage focal : 16 questions

C.7. Les phrases interrogatives: 11 questions

C.8. Les phrases exclamatives: 4 questions

C.9. Laccord numéral verbal de longue distance : 8 questions

C.10. Les phrases subordonnées conjuguées : 173 questions

- complétives : 52 questions

- relatives : 22 questions

- comparatives : 20 questions

- consécutives : 4 questions

- temporelles : 9 questions

- causales : 6 questions

- conditionnelles : 5 questions

- concessives : 4 questions

- finales : 3 questions

- objet des phrases transitives nominalisées : 3 questions

- phrases nominalisées sujet de phrases transitives: 3 questions

- syntaxe des verbes aspectuels : 9 questions

- syntaxe des verbes de perception : 3 questions

- syntaxe des verbes de connaissance : 6 questions

- syntaxe des verbes d'interaction : 9 questions

- yntaxe des verbes de réanalyse (modaux) : 8 questions

- exception exclusive : 3 questions

- verbes causatifs : 4 questions

C.11. Les phrases impersonnelles : 9 question

D. Morphophonologie (hiatus : thème nominal vocalique $+a$ ) : 5 questions 
Le questionnaire comprend également une partie consacrée à la phonologie. ${ }^{13}$ Pour ne pas considérablement allonger le questionnaire, il a été ciblé sur des éléments où les effets du contact des langues sont le plus susceptible de produire des effets. Trois types d'éléments ont été l'objet de questions :

- le mode de réalisation des emprunts comportant des phonèmes n'existant pas en basque : 79 questions

-la réalisation des phonèmes basques pour lesquelles les oppositions n'existent pas en français : 99 questions, concernant les groupes suivants :

- les sifflantes-chuintantes : 31 questions

- les affriquées : 11 questions

- les palatales : 13 questions

- la consonne aspirée et les occlusives aspirées : 20 questions

- les vibrantes : 24 questions

\subsection{La technique d'enquête.}

Les enquêtes ont été réalisées en tête-à-tête par questionnement direct et enregistrement immédiat, sans que les informateurs n'aient été préalablement informés du contenu des questions. Elles ont été menées par deux linguistes post-doctorantes, signataires de cet article, disposant d'une solide expérience en matière de recueils de données de terrain dans le domaine basque (en PBA), au cours de trois séances d'une heure et demie en moyenne. Ces dernières ont eu lieu, au choix des locuteurs, à leur domicile, ou dans les locaux du centre IKER à Bayonne. Les locuteurs ont été préalablement informés des objectifs de l'enquête et de l'usage qui serait fait des enregistrements, et chacun a signé un document écrit indiquant son accord de participation selon les procédures fixées en matière éthique (Baude 2006). Dans la base de données, les informateurs sont anonymisés, mais leurs réponses sont directement audibles, sans altération de la voix.

Eu égard à la longueur (quatre à cinq heures au total) et à la difficulté de nombreuses questions (morphologie verbale, syntaxe de subordination), l'enquête proprement dite a été précédée de tests de faisabilité, d'abord auprès de locuteurs ayant une certaine aisance linguistique (bertsolaris, étudiants avancés), puis de locuteurs ordinaires de tous âges, au total 16 locuteurs. En effet, il n'était pas évident a priori que la méthode d'interrogation directe par traduction immédiate puisse donner des résultats satisfaisants, ou soit même réellement possible. En fait, les tests de faisabilité d'abord, puis l'expérience proprement dite, ont été très positifs, et pratiquement tous les informateurs ayant donné leur accord de principe ont ensuite répondu au questionnaire dans sa totalité. ${ }^{14}$

13.- Cette partie a grandement bénéficié de la collaboration de M. Oñederra, et des observations de JI Hualde. 14.- Il convient de souligner la remarquable disponibilité des informateurs sollicités, sans lesquels cette recherche n'aurait pu être menée. 
Les conditions de réalisation de l'enquête doivent être prises en compte à l'heure de l'interprétation des données. Comme indiqué haut, chaque item du questionnaire a été élaboré pour répondre à un point précis de morphosyntaxe, et la base de données est construite en conséquence. Dans le cas de questions plus difficiles (surtout hors contexte), bien que le locuteur ne fût pas informé du but de la question, parfois l'informateur mobilise son attention sur ce point, et néglige les autres éléments de la phrase traduite. Il peut également arriver, notamment dans le cas des phrases complexes, que les informateurs aient tendance à traduire par séquences infraphrastiques, avec une perte relative des connexions entre ces séquences. Les enquêtrices de façon générale ont eu pour objectif que l'information recueillie reflète la compétence du locuteur concernant le point demandé ; par conséquent, elles n'ont pas fait confirmer par l'informateur sa réponse lorsque celle-ci s'éloignait de la phrase d'élicitation sans que cela n'affecte le point objet de la question. Il convient par conséquent de ne pas sur-interpréter et de traiter avec prudence les données résultant de ces effets liés au mode d'élicitation. Par exemple, la question C320 porte sur l'expression de l'impersonnel ('On me parle toujours comme si j'étais un enfant'). Dans la réponse les informateurs ne traduisent pas nécessairement la phrase comparative de façon stricte (Haur bati bezala bethi mintzatzen ziztatzüt répond par exemple YOSAN, 'soit 'On me parle comme à un enfant'). La réponse est considérée comme pleinement donnée, puisque l'informateur a exprimé en basque l'impersonnel. A l'inverse, on ne saurait tirer d'enseignement de cette réponse concernant une difficulté à utiliser les paradigmes du suppositif irréel chez ce locuteur.

D'autres fois, les choses peuvent être plus délicates. Par exemple, dans des phrases relativement simples portant sur un point de morphologie particulier comme le prolatif (A33 : 'Peio m'a pris pour un imbécile'), sur l'ensemble des réponses, deux informateurs n'ont pas marqué le sujet avec l'ergatif. ${ }^{15}$ Ces deux informateurs (PEOLO, MIMI) appartiennent au groupe des plus jeunes, et ils ont le français comme première langue. Il peut s'agir d'une simple erreur, ou d'un effet du mode d'élicitation comme précédemment (bien que la brièveté de la séquence ne pousse pas à penser cela). Comme dans les questions visant spécifiquement l'ergatif, tous les locuteurs, sans exception, ont utilisé la marque, seul un examen plus approfondi des réponses de ces informateurs peut indiquer si ceci correspond à un usage affaibli de l'ergatif pour ce type de locuteurs. Ce n'est pas le seul exemple de ce type, loin s'en faut. ${ }^{16}$

Un autre point, enfin, doit être précisé concernant la technique de transcription des réponses. Après beaucoup d'hésitations, la solution adoptée a été celle de la transcription intermédiaire. La base de données étant sonore et extrêmement facile d'accès, la transcription phonétique, qui aurait eu un coup en temps très important car il eut fallu l'accompagner en

15.- Le cas inverse (marquage ergatif dans un contexte où il est exclu) est également attesté de façon plus rare. Des exemples d'un tel emploi apparaîssent dans les réponses recueillies dans cet article, ainsi en 3.1.3. (note 24) et en 3.2.3. (note 26).

16.- Voir par exemple, en 3.1.3, la dernière réponse (iriarantz) du groupe d'âge intermédiaire concernant l'emploi de l'adlatif approximatif, qui correspond cependant peut-être à une sur-correction sur la forme régulière irirantz). 
pratique d'une transcription en graphie ordinaire, n'a pas paru indispensable. Une transcription en langue proche des standards écrits (même dialectaux) a été envisagée, mais il a semblé que dans bien des cas cela aurait conduit à rendre plus long le travail des chercheurs, lesquels, généralement, effectuent une première approche des données à partir des transcriptions, avant d'approfondir en recourant aux enregistrements oraux. La transcription orthographique semiphonologique, très répandue d'ailleurs dans les études basques, implique une certaine variation selon les auteurs des transcriptions, car il vise à concilier l'impression acoustique et l'interprétation à travers les codes semi-orthographiques. Bien sûr, divers inconvénients résultent de cette technique, mais ils ont paru moindres que ceux qui auraient résulté d'autres choix, d'autant qu'il n'y a eu que deux transcripteurs, chaque enquêtrice ayant transcrit les données qu'elle a recueillies. Mais, ici encore, il faut éviter les sur-interprétations et se reporter au matériel sonore. Par exemple la réponse de l'informatrice souletine YOSAN à la question C317 ('On parle en quelle langue dans cette ville?') est transcrite Zer mintzaje emaiten di(e) herri huntan? La parenthèse indique que le morphème qui y est inséré est inaudible. Pourtant, on ne peut conclure de cette transcription une quelconque chute de l'indice d'ergatif final dans la forme verbale souletine díe (standard dute) chez cette locutrice; il s'agit d'une réalisation dans un contexte où le verbe die (accent sur la première voyelle) est suivi sans pause d'une séquence à initiale en he-

\section{Quelques changements linguistiques observés dans la base de données.}

On peut distinguer trois principaux types de changements linguistiques pouvant affecter les parlers basques d'Aquitaine dans des usages non familiers : ${ }^{17}$

- ceux résultant d'une influence du basque standard ou commun se traduisant par une convergence par rapport au reste des parlers basques;

- ceux résultant d'une influence du français se traduisant par une divergence par rapport au reste des parlers basques;

- ceux résultant simplement d'une évolution interne, non directement attribuable au contact.

Pour illustrer le type d'enseignement que l'on peut retirer de la base de données NoRANTZ, nous allons donner quelques exemples simples de ce type de changements tels qu'ils peuvent être observés dans la base de données NoranTz.

17.- Les conditions de réalisation de l'enquête conduisent à des données correspondant plutôt à un registre de langue non familier (traductions sollicitées par des enquêtrices bascophones, au départ inconnues et natives d'un autre parler). Les données de la base de données ne doivent pas conduire à préjuger de l'emploi ou non des formes plus dialectales que celles recueillies dans les usages familiers de la langue. 


\subsection{Changements de convergence.}

Ces exemples sont très nombreux, ce qui n'est pas étonnant compte tenu du développement du basque standard et du poids important pris par les locuteurs d'outre-Bidassoa dans la production culturelle et médiatique en langue basque. Nous allons l'illustrer ici à travers des réponses recueillies concernant les points suivants relevant de la morphologie casuelle, la morphologie verbale, et d'un marqueur de connection de subordination, soit, plus précisément:

i) la forme de la désinence casuelle de datif pluriel;

ii) la racine de l'auxiliaire avec les verbes ditransitifs tripersonnels au présent;

iii) la réalisation de l'adlatif approximatif;

iv) le suffixe des complétives sélectionnées par des verbes de phrases négatives.

\subsubsection{Le datif pluriel}

La désinence de datif pluriel est -ei en basque standard, et -eri (occidental) ou -er (oriental), dans les dialectes d'Aquitaine. En distinguant les réponses fournies par les informateurs à la question visant ce trait morphologique (A21 : La mère a donné le livre aux enfants), on obtient les réponses suivantes par groupes d'âge :

\section{Informateurs âgés (+65 ans):}

Amak eman diote liburua aurreri.

Liburu haurrer eman du amak.

Amak eman diote aurreri eta... liburua.

Amak eman du liburu bat aurreri.

Amak eman titzü libüiak haurrer.

Amak eman du liburua haurrei.

Amak eman du liburia haurrer.

Amak eman du liburua aurreri.
Amak eman du liburua haurreri.

Amak eman dizü lübüia haurrer.

Amak eman dü libria haurrer.

Amak liburua eman du aurreri.

Amak eman du liburia haurreri.

Amak eman dü libria haurrei.

Amak eman du liburia bere haurrer.

- Désinences de datif pluriel réalisées sur haur- 'enfant': -ei : 02; -eri : 07; -er : 06

Comme, on le voit, chez les locuteurs les plus âgés, la plupart des locuteurs (13/15) utilisent une forme dialectale (-eri ou -er) dans leur réponse.

Le groupe des informateurs d'âge intermédiaire (40-60 ans):

Amak eman ditu liburuak aurreri.

Amak liburua eman die aurreri.

Amak eman dizü libria haurrer.

Amak eman dü libria haurrer.

Amak liburua eman du haurrer.

Amak liburua eman die haurrer.
Amak eman du liburua aurreri.

Amak eman du liburua haurrer.

Amak liburua haurreri eman dakote.

Amak eman du liburua aurrer.

Amak eman die liburua aurrei.

Amak eman du liburua aurreri. 
- Désinences de datif pluriel réalisées sur haur- : -ei : 01; -eri : 05; -er : 06

Les réponses des informateurs du groupe d'âge intermédiaire sont globalement semblables à celles des locuteurs les plus âgés, la forme la plus standardisée (-ei) n'étant employée que par un seul d'entre eux (1/12).

Le groupe des informateurs jeunes (-30 ans) scolarisés en immersion:

Amak liburua eman die aurrei.

Amak aurrer eman die liburua.

Amak liburua eman die aurrei.

Amak liburua eman die aurrei.

Amak liburua eman du aurrei.

Amak aurrei liburua eman du.

Amak liburua eman dee aurrei.

Amak liburua eman die aurrei.
Amak liburua eman die aurrei.

Amak eman du liburua aurrei.

Amak liburua eman du aurrei.

Amak liburua eman du aurrei.

Amak liburua eman du aurrei.

Amak aurrei liburua eman die.

Amak liburua aurrei eman du.

Amak haurrei liburua eman die.

Amak aurrei liburua eman die.

- Désinences de datif pluriel réalisées sur haur- : - ei : 15; -eri : 00; -er : 01

Les réponses des locuteurs les plus jeunes scolarisés en basque font apparaître un réel changement dans les usages non familiers, corrrespondant à celui de l'enquête, puisque la forme standard y supplante presque entièrement les formes dialectales.

Le groupe des informateurs jeunes (-30 ans) scolarisés hors immersion:

Amak liburua aurrer eman diete.

Amak liburua eman die aurrei.

Amak liburua eman dizkie aurrei.

Amak dizü libriak haurrer.

Amak liburua eman du aurreri.

Amak eman die liburua aurrei.

Amak eman tütü libriak haurrer.
Ama (sic) eman dizü libria haurrer.

Amak liburua eman die aurrei.

Amak haurrer liburia eman dee.

Amak eman du liburua aurreri.

Amak eman dizü libria haurrei.

Amak eman dizü liburrua haurrei.

Amak aurrei liburua eman die.

- Désinences de datif pluriel réalisées sur haurr- : - ei : 07; -eri : 02; -er : 05

Les réponses recueillies chez les jeunes qui ne sont pas scolarisés en mode bilingue reflètent également la forte présence de la désinence standard (07/14), même si une moitié des réponses correspond aux formes dialectales.

La base de données ne permet pas de traitement statistique, car les locuteurs n'ont pas été sélectionnés sur des critères autorisant une telle approche. Il n'en demeure pas moins que les éléments recueillis indiquent une claire évolution relative à la réalisation de la désinence du datif pluriel en situation d'enquête formelle. Chez les informateurs des deux groupes les plus âgés, la forme du basque standard est très minoritaire, et elle n'a été utilisée que par des informateurs bien alphabétisés lisant régulièrement en basque. Par contre, chez le groupe des 
jeunes, c'est la réponse de loin la plus fréquente, de façon presque unanime chez les jeunes scolarisés dans le système d'immersion, et de façon significative chez ceux n'ayant pas eu une scolarisation bilingue en immersion. Les locuteurs les plus jeunes ont spontanément donné la réponse qui leur paraissait la plus naturelle dans un contexte non familier, comme celui du questionnaire. Comme souligné précédemment, ceci ne préjuge évidemment pas de la forme du suffixe de datif pluriel que ces mêmes informateurs utilisent dans leur famille ou leur environnement proche.

\subsubsection{L'auxiliaire des formes verbales tri-personnelles ditransitives.}

La morphologie verbale représente un élément important du questionnaire NORANTZ. Plusieurs questions concernant la réalisation des formes tri-personnelles y sont incluses, en fonction de paramètres différents (temps, mode, jeu des personnes). ${ }^{18}$ Parmi les formes les plus emblématiques permettant de repérer une tendance de convergence chez les locuteurs, nous avons retenu celle du présent impliquant les personnes de dialogue dans des phrases ditransitives.

Dans les parlers traditionnels, des phénomènes de divergence par rapport aux parlers occidentaux existent depuis longtemps. Certains sont fort anciens, comme celui de l'optionalité de l'accord datif dans de nombreux parlers orientaux, ou celui relatif à l'usage d'une racine d'auxiliaire identique à celle des formes monotransitives $(-(d) u-)$. Ces deux éléments étaient déjà présents au XVIème siècle. D’autres sont plus récents, comme celui correspondant à un réaménagement de la place de l'indice personnel d'accord datif. Développé principalement dans la seconde partie du XIXème siècle dans les parlers labourdins (comme d'ailleurs le long de la côte au-delà de la frontière), ce réaménagement consiste à préfixer les indices personnels de datifs (suffixés dans les formes non divergentes).

Parmi ces éléments, nous avons retenu ici celui directement lié au développement du basque standard, à savoir celui lié au choix de la racine verbale de l'auxiliaire des verbes ditransitifs, et plus précisément l'emploi éventuel de -i-. Cet auxiliaire n'est pas utilisé dans les dialectes aquitains traditionnels d'Aquitaine, sauf, pour les parlers les plus occidentaux, lorque le datif est de $3^{\text {ème }}$ personne, mais jamais avec des datifs de $1^{\text {ère }}$ ou $2^{\text {ème }}$ personne. Il est donc intéressant d'observer les réponses à une question simple élicitant l'emploi d'un verbe ditransitif au présent, avec des arguments ergatif et datif de lère et 2ème personnes. Une question visant ce point est B79 : Vous m'avez donné le livre de mon frère. Voici les réponses, qui nous permettront de voir dans quelle mesure les informateurs ont employé l'auxiliaire standard en -i- dans leurs réponses, en situation d'enquête.

Informateurs âgés (+65 ans):

Eman nauzu nere anaien liburua.

Ene anaian liburua eman dautazu. 
Nere anaiaren liburua eman nauzu/dautazu.

Eman nauzu liburua ene anaiaina.

Eman nauzu nere anaien liburua.

Eman deitazüt ene anaiae(n) libüia.

Eman dautazu ene aneiaren liburua.

Eman dautazu ene anaian liburüa.

Nere anaiaren librua eman didazu.
Eman deitaziet anaien lübüia.

Eman deitazüt ene anaian libria.

Anaiaren liburua eman dautazut.

Eman dautazu ene anaiaren liburua.

Eman deitadazüt ene anaian libria.

Eman dautazu ene anaiaren liburua.

- Radical -i- : 01/15

Chez le groupe d'informateurs les plus âgés, les réponses sont proches de celles attendues pour les locuteurs reflétant le mieux les usages traditionnels, avec cependant l'absence complète de formes conjuguées sans accord datif. ${ }^{19}$ Conformément aux attentes, les informateurs de parler labourdin (du moins 3 sur 4) ont employé une forme verbale où le datif est préfixé (nauzu), sans 'corriger' une forme souvent considérée comme fautive (un informateur a proposé les deux formes, mais il s'agit d'un locuteur de parler bas-navarrais). Un seul informateur a utilisé la forme verbale du basque standard : il s'agit d'une informatrice ayant vécu longtemps hors du Pays Basque, et qui, à son retour, a vécu dans un environnement bascophone varié.

Le groupe des informateurs d'âge intermédiaire (40-60 ans):

Ene anaien liburua eman nauzu.

Eman didazue nere anaiaren liburua.

Eman deitazüt ene anaiae(n) libria.

Ene anaien libria eman deitaziet.

Ene anaiaren liburua eman dautazu.

Ene anaiaren liburua eman dautazu.
Eman datazie ene anaiaren liburua.

Ene anaiaren liburua eman didazu.

Nere anaiaren liburua eman dautazu.

Eman dautazu nere aneiaren liburua.

Eman didazu ene anaiaen liburua.

Ene anaiaren liburua eman didazu.

- Radical -i- : 04/12

Les résultats chez les locuteurs d'âge intermédiaire sont logiquement dans le prolongement de ceux du groupe le plus âgé. L'accord datif est marqué dans toutes les réponses, l'usage

19.- Cette option existe dans les parlers ici étudiés, mais elle n'est pas mise en œuvre par les informateurs dans cette question. Il est difficile de savoir s'il s'agit d'un effet de convergence rendant cette option de moins en moins disponible, ou si cela résulte de la question elle-même et du mode d'élicitation, qui, tous deux, favorisaient la mise en jeu des accords. Les réponses données dans d'autres questions portent à penser que la seconde option est la plus probable. On remarquera, par exemple, que dans les réponses à la question A21 ciblant les formes de la désinence de datif pluriel et rapportées plus haut (3.1.1) de nombreuses réponses ne font pas apparaître l'accord datif dans un contexte où le syntagme datif est réalisé hors du verbe. Cet accord y est nettement minoritaire dans les deux groupes les plus âgés (2/15 et 4/12), et légèrement majoritaire (10/17), ou égal (07/14), aux cas de non accord dans les deux groupes de jeunes. 
de l'auxiliaire à radical -i- est un peu plus présent, mais nettement minoritaire (03/12) ${ }^{20}$

Le groupe des informateurs jeunes (-30 ans) scolarisés en immersion:

Nere anaiaren liburua eman nauzu.

Ene anaiaren liburua eman didazue.

Eman nauzue nere anaiaren liburua.

Nere anaiaren liburua eman didazu.

Nere anaiaren liburua eman didazu.

Nire anaiaren liburua eman didazu.

Ene anaiaren liburua eman ditazie.

Nere anaiaren liburua eman didazu.

Nere anaiaren liburua eman didazu.
Nere anaiaren liburua eman didazu.

Ene anaiaren liburua eman didazu.

Nere lagunaren liburua eman dautazu.

Nere anaiaren liburua eman didazu.

Eman didazue ene anaiaren liburua.

Nere anaiaren liburua eman didazute.

Nere anaiaren liburua eman didazue.

Nere anaiaren liburua eman didazu.

\section{- Radical -i- : 13/17}

Chez les locuteurs du groupe des jeunes ayant suivi une scolarisation en basque, les résultats sont très différents. Lauxiliaire en -i- est utilisé dans la grande majorité de leurs réponses, ces informateurs, dans le contexte de l'enquête, privilégiant la forme standard (13/17). ${ }^{21}$

Le groupe des informateurs jeunes (-30 ans) scolarisés hors immersion:

Ene anaiaren liburua eman dautazu.

Nire anaiaren liburua eman didazue.

Nere anaiaren liburua eman didazue.

Nere anaiaren liburua eman didazu.

Eman didazue nire anaiaren liburua.

Eman deitazüt ene anaian libria.

Anaiaren liburua eman didazu.
Eman deitazüt ene anaian libria.

Nere anaiaren liburua eman didazue.

Ene aniain liburia eman daazi.

Ene anaian libria eman deitaziet.

Sans réponse recueilllie

Ene anaiaren liburua eman didazue.

Eman düzü ene enaien librua.

- Radical -i- : 07/13

Chez les informateurs jeunes n'ayant pas suivi une scolarisation en basque, l'emploi de la forme verbale en -i- est moins présente - logiquement - que dans le groupe précédent, mais elle est malgré tout très remarquable.

20.- Sur les deux locuteurs natifs d'un parler labourdin, un seul a utilisé la forme à préfixe datif.

21.- Les formes à datif préfixé $(02 / 17)$ sont présentes, quoique très minoritires $(02 / 17)$, ce qui montre la persistance, au moins marginale, de ce phénomène chez ces jeunes locuteurs, malgré sa stigmatisation dans le contexte scolaire. 
Les données relatives à l'auxiliaire des formes ditransitives tripersonnelles sont largement parallèles à celles observées précédemment pour la morphologie de la désinence de datif pluriel. En situation d'enquête, les formes standard sont largement représentées chez les locuteurs les plus jeunes, y compris, mais à un degré bien moindre, chez ceux qui n'ont pas reçu une scolarisation bilingue avec immersion en basque. Rappellons, par ailleurs, que l'usage des formes standard chez les jeunes informateurs, peut correspondre a deux situations distinctes : celle où il s'agit pour eux d'une forme optionnelle, marquant un type d'usage différent des emplois familiers de la langue, et celle où il s'agit de la seule forme disponible pour eux, en particulier lorsque leur compétence en basque a été acquise essentiellement dans le cadre scolaire.

\subsubsection{L'adlatif approximatif.}

La morphologie des suffixes casuels varie relativement peu selon les dialectes. Cette variation peut être plus marquée dans certaines formes complexes, où, selon les dialectes, des postpositions différentes se joignent à un autre suffixe, donnant lieu éventuellement à la création d'une forme figée. C'est le cas pour l'adlatif approximatif (dit aussi directionnel) qui correspond à la préposition vers du français. Cet adlatif approximatif est exprimé par le suffixe -rantz, qui est au départ uniquement d'usage occidental et inconnu dans les dialectes traditionnels d'Aquitaine,$^{22}$ ou par une forme synonyme non synthétique, utilisée dans les dialectes du PBA, avec un morphème autonome (buruz ou alde) sélectionnant un syntagme adlatif (et datif également pour buruz). Le basque standard autorise les deux formes de cet adlatif, et par conséquent, l'usage du suffixe synthétique chez les locuteurs aquitains fait apparaître une convergence, avec extension vers les dialectes d'Aquitaine d'une forme inconnue d'eux jusquelà. La question destinée à tester ce point dans la base de données NORANTZ est relativement complexe, car elle inclut une phrase complétive subjonctive au passé (sur ce sujet, voir infra 3.2.3). Il s'agit de A47: Je ne voulais pas que Maddi aille vers le village. ${ }^{23}$ Certains des informateurs ont répondu en utilisant la forme adlative simple, sans traduire strictement la phrase d'élicitation sur ce point. Ces réponses sont présentées ci-dessous (le morphème locatif n'y est pas mis en gras), mais elles ne sont pas prises en compte dans l'analyse, car elles ne permettent pas de mettre en évidence un choix entre leux types d'adlatif approximatif.

Le point ici est donc de savoir dans quelle mesure les informateurs utilisent une forme issue du basque socialement le plus dynamique, inconnu dans leur parler traditionnel, sans que les règles du basque standard n'obligent à son emploi. Comme nous allons le constater, le contraste est très net entre les différentes générations d'informateurs.

Informateurs âgés (+65 ans):

22.- Le suffixe -rantz est issu de -ra+ontz. Pour une analyse étymologique de cette formation, voir Gómez (2005).

23.- On remarquera dans les réponses que de nombreux informateurs ne réalisent pas la concordance temporelle dans la subordonnées (possiblement un effet du contact des langues, le français ayant largement perdu l'opposition passé/présent dans ce contexte). 
Ez nuen nai Maddi errirat joaitia.

Ez nuen nahi Maddi herri alderat joan tzadin.

Ez nuen nai Maddi doan tzain eta... iriat.

Enuen nai Maddi joan tzadin errirat.

Enikezün nahi Maddi jun dadin kharrikako aldilat.

Enuen nahi joaitia Maddi hirirat.

Enuke nahi Maddi joan dadien herriari buruz.

Ez nuen nai Maddi erriari buruz joan zadin.
Ez nuen nahi Maddi joan zain herri horri buruz.

Enizün nahi Maddi jun ledin kharrikalat büüz.

Enian nahi Maddi jun ledin kharrikalat büüz.

Enuen nai Maddi joan dadin errirat.

Ez nuen nai Maddi juan zadin erri alderat.

Enian nahi Maddi joan dadin kharrikarat büüz.

Enian nahi Maddi joan zain herriari buruz.

- Usage du suffixe -rantz:00/10 (+5 réponses avec adlatif simple)).

Dans le groupe des locuteurs les plus âgés, aucune réponse ne présente le morphème -rantz.

Le groupe des informateurs d'âge intermédiaire (40-60 ans) :

Enuen nai Maddi irixkaren alde joan dadin.

Enuen nai Maddi joan zedin errira.

Enüzün nahi Maddi jun ledin kharrikalat.

Enizü nahi Maddi jun dadin herrilat büz.

Ez nuen nahi Maddi joan tzain herriari buruz.

Ez nuen nahi Maddi herri alderat joan tzadin.
Enuen nai Maddi erriari buruz juaitea.

Enuen nai Maddi erriruntz joan dadin.

Enuen nahi Maddi joan tzadin herrirat.

Ez nuen nahi Maddi joan zain herrira buruz.

Ez nuen nai Maddi joan dadin erriaren buruz.

Ez nuen nai Maddi joitea iriarantz. (sic) ${ }^{1}$

\section{- Usage du suffixe -rantz : 02/09 (+3 adlatifs simples)}

Chez les locuteurs d'âge intermédiaire, seules deux informateurs ont eu recours au suffixe-rantz (ou sa variante-runtz).

Le groupe des informateurs jeunes (-30 ans) scolarisés en immersion:

Ez nuen nai Maddi errirantz joatea.

Ez nuen nahi Maddi errira buruz joan tzedin.

Ez nuen nai Maddi joatea erriruntz.

Enuen nai Maddi errirantz joan tzedin.

Ez nuen nai Maddi joaitea erriruntz.

Ez nuen nai Maddi joan dadin errira buruz.

Ez nuen nai Maddi errirantz joan tzedin.

Ez nuen nai Maddi errirat joan dadila.

Ez nuen nai Maddi joan zedin errixkaruntz.
Enuen nai Maddi erriruntz joan dadin.

Ez nuen Maddi erriruntz joatea nai.

Ez nuen nai Maddi erriruntz joan dadin.

Ez nuen nai Maddi erriruntz joan dadin.

Ez nuen nai Maddi errirat joaitea.

Ez nuen nai Maddi errirat joatea.

Ez nuen nai Maddi iriruntz joaitea.

Enuen nai Maddi juatea erriruntz.

- Usage du suffixe -rantz: $12 / 14$ (+3 adlatifs simples)

Le groupe des informateurs jeunes (-30 ans) scolarisés hors immersion: 
Ez nuen nai Maddi herrira buuz joan dadin.

Ez nuen nai Maddi errirantz joatea.

Ez nuen nai Maddi joaitea erriruntz.

Enuen nai Maddi errirat joan zedin.

Ez nuen nai Maddi errirantz joan dadila.

Enizün nahi Maddi jun ledin kharrikalat büüz.

Enuen nai Maddi errirantz joatea.
Enizün nahi Maddi jun dadin kharrikala büüz.

Ez nuen nai Maddi erriruntz joatea.

Enin nai Maddi errirat joan tzain.

Eniz (sic) nahi Maddi jun dadin herrilat büz.

Enizü nahi Maddi herrilat büz jun ledin.

Ez nuen nai Maddik (sic) errira buruz juaitea. ${ }^{2}$

Enizün nahi Maddi jun tzen herringanat (sic).

- Usage du suffixe -rantz: 05/12 (+ 2 adlatifs simples)

Ainsi qu'on peut le constater, le suffixe -rantz est utilisé par une nette majorité des jeunes scolarisés en basque, et près de la moitié des autres. Le groupe des informateurs les plus âgés n'est donne aucun témoignage, et ceux d'un âge intermédiaire, fort peu. Comme indiqué précédemment, le basque standard intègre tout aussi bien les formes synthétiques (-rantz) que non synthétiques (avec la postposition buruz) pour l'expression de l'adlatif approximatif. Il s'agit donc d'une convergence résultant, non des prescriptions du basque standard académique, mais plutôt de l'influence des dialectes, socialement les plus forts, d'outre-Bidassoa. On a la confirmation de ce fait, en observant que le suffixe est souvent réalisé sous une forme dialectale du suffixe : -runtz, et non dans sa forme standard -rantz.

Un autre exemple de cette influence de ces dialectes se retrouve dans le trait ci-dessous, qui concerne non plus la morphologie casuelle, mais la morphologie de la subordination.

\subsubsection{Le suffixe des complétives sélectionnées par des verbes de phrases négatives.}

En basque, la morphologie de subordination est essentiellement affixale. Dans les phrases complétives, elle est réalisée dans tous les dialectes par le suffixe -(e)la, qui se joint au verbe de la phrase complétive déclarative. Néanmoins, depuis deux siècles environ, les dialectes occidentaux ont développé un suffixe de complétive spécifique, utilisé lorsque le verbe sélectionnant la complétive est dans une phrase négative (EGLU-V 1999:73). En dehors de quelques cas spécifiques, ce suffixe -(e)nik, apparenté au partitif, est devenu aujourd'hui d'usage général dans ces dialectes. Le basque standard accepte les deux suffixes - (e)la et -(e) nik dans les contextes négatifs, et par conséquent les cas d'expansion du suffixe-(e)nik dans les parlers du PBA reflètent surtout une influence des dialectes centraux et occidentaux plutôt que celui des normes de standardisation académique. Voyons comment ce point apparaît dans la base de données NORANTz à travers les différents groupes d'informateurs. Nous le ferons à travers la question C150: Maddi n'a pas dit qu'elle viendra demain).

Informateurs âgés (+65 ans):

Maddik eztu erran biar etorriko dela.

Maddik ez du erran biar etorriko dela.

Maddik eztu erran biar eldu dela.

Maddik eztu erran biar etorriko dela.
Maddik eztu erran bihar jinen dela.

Maddik eztizü erran bihar jinen zela.

Maddik eztü erran bihar jinen dela.

Maddik eztu erran etorriko dela biar. 
Maddik eztizü erran bihar jinen dela. Maddik eztu erran biar jinen dela.

Maddik etzuen erran etorriko zela biar. Maddik eztü erran bihar jinen zela.

Maddik eztu erran bihar ethorriko dela. Maddik eztu erran bihar jinen dela.

Maddik eztu erran biar etorriko dela.

- Usage du suffixe -(e)nik : 00/15

Dans le groupe des locuteurs les plus âgés, aucun informateur n'a utilisé le suffixe spécifique des complétives de verbe au négatif des dialectes occidentaux.

Le groupe des informateurs d'âge intermédiaire (40-60 ans):

Maddik eztu erran biar etorriko zela.

Maddik eztu erran biar etorriko zela.

Maddik eztizü erran biar jinen zela.

Maddik eztizü erran bihar jinen lizatekila.

Maddik ez du erran bihar jinen dela.

Maddik eztu erran biar jinen dela.
Biar etorriko dela, eztu erran Maddik.

Maddik eztu erran biar etorriko zela.

Maddik ez du erran biar etortzen zela.

Maddik eztu erran bihar jinen zela.

Maddik ez du erran biar etortzen dela.

Maddik erran dit etorriko dela biar.

- Usage du suffixe -(e)nik : 00/12

Dans le groupe des informateurs d'âge intermédiaire, comme dans celui des plus âgés, nul informateur n'a utilisé le suffixe spécifique des complétives de verbes au négatif.

Le groupe des informateurs jeunes (-30 ans) scolarisés en immersion:

Maddik eztu erran biar etorriko zela.

Maddik eztu erran biar etorriko denik.

Maddik ez du erran biar etorriko dela.

Maddik eztu erran biar etorriko denik.

Maddik ez du erran biar etorriko zela.

Maddik ez du erran biar jingo zela.

Maddik eztu erran biar jinen denik.

Maddik eztu erran biar etortzeko zenik.
Maddik ez du erran biar jinen zela.

Maddik eztu erran biar etorriko zenik.

Maddik eztu erran biar etorriko dela.

Maddik eztu erran biar eldu dela.

Maddik eztu erran biar etorriko zenik.

Maddik ez du erran biar etorriko zenik.

Maddik ez du erran biar etorriko dela.

Maddik erran dit biar etorriko zela.

\section{- Usage du suffixe -(e)nik : 07/17}

Dans le groupe des jeunes scolarisés en basque la présence du suffixe -(e)nik est marquée, même si elle est loin d'être généralisée, et la comparaison avec d'autres questions où ce type de données est présent montre que l'emploi du suffixe reste uniquement optionnelle pour la plupart de ces informateurs. Probablement un marqueur de registre soigné supradialectal. 
Le groupe des informateurs jeunes (-30 ans) scolarisés hors immersion:

Maddik ez du erran biar jinen dela.

Maddik ez du erran biar etorriko dela.

Maddik ez du erran biar etorriko zenik.

Maddik eztu esan biar etorriko dela.

Maddik ez du erran biar etorriko zela.

Maddik eztizü erran bihar horra zela.

Maddik eztu erran biar etorriko zela edo biar etorriko zenik.
Maddik eztizü erran jinen zela bihar. Maddik eztu erran biar eldu zenik. Maddik eztu erran biar etorriko dela. Maddik eztizü erran bihar juiten tzela. Maddik eztizü erran bihar jinen dela. Maddik ez du erran etorriko zela biar. Maddik eztizü erran jinen tzela bihar.

- Usage du suffixe-(e)nik: 03/14

Dans le groupe des jeunes non scolarisés en basque le suffixe -(e)nik est présent, mais de façon moins marquée que dans le groupe précédent.

Les données recueillies ici sont en complète harmonie avec celles observées plus haut portant sur le suffixe d'adlatif approximatif. Le basque standard normalisé admettant l'usage des dialectes d'Aquitaine, lesquels emploient le suffixe -(e)la, et non -(e)nik, dans ces complétives, le recours à ce dernier morphème par ces locuteurs lors de l'enquête fait apparaître une influence des dialectes d'outre-Bidassoa dans un tel contetxe, plus qu'un effet direct des prescriptions du basque standard. On observera que ceci est plus sensible chez les informateurs les plus jeunes, et notamment ceux scolarisés en basque. Cependant l'emploi du suffixe spécifique -(e)nik est également présent parmi les jeunes locuteurs qui ne sont pas scolarisés en basque.

\subsection{Exemples de changements de divergence.}

Si beaucoup des changements repérables par ce type d'enquêtes correspondent, comme dans les cas précédents, à des phénomènes de convergence, la base de données Norantz donne aussi la possibilité de repérer des phénomènes de divergence, parfois, quoique pas toujours, attribuables à un effet du contact avec le français. Ces derniers phénomènes sont particulièrement perceptibles dans le domaine phonétique, mais ils peuvent apparaître également, de manière plus subtile, dans celui de la morphosyntaxe.

Nous n'évoquerons pas ici les données touchant à la phonétique, qui sont bien connues dans leurs aspects les plus caractéristiques : réalisation uvulaire du phonème $[R]$, tendance à amuïr les nasales dentales en coda avant consonne (avec conservation du trait de nasalité sur la voyelle), maintien de phonèmes non basques dans de nombreux termes empruntés, etc...). Nous présenterons les données relatives aux points suivants:

i) le marquage casuel du causee dans les formes causatives de certains verbes intransitifs; 
ii) l'emploi du réflexif lexical;

iii) l'emploi du passé dans les phrases complétives subjonctives;

iv) l'usage de l'adlatif avec un verbe de mouvement.

\subsubsection{Le traitement du causé dans les formes causatives des verbes intransitifs à} verbe léger transitif.

Les formes verbales causatives sont réalisées en basque par l'emploi du suffixe -arazi qui se joint au radical verbal du verbe causativé. Il s'agit d'une forme causative synthétique dans laquelle les arguments du verbe de base sont l'objet d'un réalignement casuel et syntaxique consécutif à l'intégration de l'argument majeur du causatif. Lorque le verbe subissant la causation est un verbe intransitif (dont le sujet en général est à l'absolutif en basque), l'argument y correspondant dans la forme causative (dit causé) reste à l'absolutif, ${ }^{24}$ tandis que le sujet du verbe causatif est à l'ergatif. Les exemples suivants illustrent ce point :

$\begin{array}{llll}\text { Jon } & \text { Maiarekin } & \text { jostatu } & \text { da } \\ \text { Jon.ABS } & \text { Maia.COM } & \text { joué } & \text { AUX }\end{array}$

'Jon a joué avec Maia.'

\begin{tabular}{|c|c|c|c|}
\hline Amak & Jon & Maiarekin & jostarazi \\
\hline mère.ERG & Jon.ABS & Maia.COM & joué \\
\hline
\end{tabular}

'Sa mère a fait jouer Jon avec Maia.'

Comme on peut le constater dans les exemples ci-dessus, le syntagme correspondant à l'argument sujet (Jon) du verbe jostatu 'jouer' est à l'absolutif dans les deux phrases : celle où il correspond à un sujet du verbe intransitif, et celle où il est l'objet direct du verbe causatif.

Dans le cas où le verbe de base auquel se joint le causatif est un verbe transitif, avec donc un argument sujet à l'ergatif, c'est l'argument correspondant à l'objet direct qui est à l'absolutif, comme dans l'exemple suivant:

$\begin{array}{llll}\text { Haurrak } & \text { sagarrak } & \text { jan } & \text { ditu } \\ \text { enfant.le.ERG } & \text { pomme.les.ABS } & \text { mangé } & \text { AUX }\end{array}$

'L’enfant a mangé les pommes.'

Dans les constructions causatives, l'argument sujet du verbe de base, autrement dit le causé, prend le cas datif, l'objet direct (sagarrak, 'les pommes') restant lui au cas absolutif,

24.- Nous ne tenons pas compte ici de la dativation de ces causés animés, option présente outre-Bidassoa, mais pas, ou marginalement, dans les parlers aquitains. 
comme illustré ci-dessous :

$\begin{array}{lllll}\text { Amak } & \text { haurrari } & \text { sagarrak } & \text { janarazi } & \text { dizkio } \\ \text { mère.la.REG } & \text { enfant.le.DAT } & \text { pomme.les.ABS } & \text { mangé } & \text { AUX }\end{array}$

'La mère a fait manger les pommes à l'enfant.'

Le basque, par ailleurs, construit une série de verbes sémantiquement inergatifs par une forme composé associant un nom simple du type eztul 'toux', negar 'pleur', lan 'travail', lo 'sommeil', etc. au verbe léger transitif egin 'faire', pour exprimer des procès intransitifs (inergatifs) correspondant aux verbes français 'tousser', 'pleurer', 'travailler', 'dormir', ... Dans la langue classique (et standard), dans tous les dialectes, lorsque ces verbes sont causativés, ils suivent le régime des verbes transitifs, mais s'agissant de verbes sémantiquement intransitifs, où par ailleurs le statut syntaxique du nom incorporé est incertain, il s'agit d'un point de morphosyntaxe susceptible de variation. Dans le cas présent, un éventuel effet du contact avec le français peut être attendu, car en français les arguments causés de verbes intransitifs du type rire, pleurer ou dormir sont traités comme des objets directs, ainsi que le montre l'emploi du clitique les dans l'exemple ci-dessous:

\section{Ces enfants, c'est vous qui les avez fait pleurer}

Le questionnaire NORANTZ a une question testant spécifiquement ce point. Il s'agit de la question C314 : Vous me faites rire, dans laquelle le marquage casuel des objets, en pratique, ne peut se faire qu'à travers la forme verbale. En effet, le basque est une langue dite 'pro-drop', où les pronoms personnels de $1^{\text {èr }}$ et $2^{2 \text { eme }}$ personnes, sujets ou objets, non emphatiques, sont indexés au sein de la forme verbale, mais omis en tant que syntaxes nominaux. Examinons les réponses, le point consistant à voir si l'indice verbal correspondant à l'argument causé est datif (basque classique et standard actuel) ou absolutif (possiblement par effet du contact avec le français). A noter cependant que pour les locuteurs de parler labourdin, qui préfixent l'indice de datif (comme l'indice d'absolutif) dans les formes verbales tri-actancielles (voir plus haut 3.1.2), il est impossible dans les réponses de déterminer le cas (absolutif ou datif) de l'argument causé.

Informateurs âgés (+65 ans):

Irriñazten nauzu.

Irriarazten nauzu.

Arek irriñazten nau.

Zuk neri irrinazten nauzu.

Erri eginazten deitazü.

Irri inaazten nauzu.
Irriaazten nauzu.

Erri eginazten deitaziet.

Erri eginaazten deitazüt.

Irri eginarazten nauzu.

Irri eginarazten daazu.

Erri eginaazten deitazüt. 
Irri inarazten nuzu.

Irrinarazten nuzu.

Irrinarazten nauzu.

- Causé préfixé : 10/15 (6 où il est absolutif, et dont 4 pour lesquels l'indice préfixé peut être datif)

- Causé suffixé (datif) : 05/15

Les résultats font clairement les deux possibilités de marquage casuel du causé dans les réponses. Même si, partiellement, les réponses montrant un causé préfixé sont ambiguës, puisque quatre informateurs sont de dialecte labourdin, on observe une forte présence des formes où l'indice de causé est préfixé, et correspond à un absolutif.

Le groupe des informateurs d'âge intermédiaire (40-60 ans) :

Irriñazten nauzu.

Irriarazten nauzue.

Erri eginazten deitazüt.

Erri eginazten deitazüt.

Irri inarazten nauzu.

Irri inaazten nauzu.
Irriazten nauzu.

Irriarazten nauzu.

Irrinazten nauzu.

Irrinaazten nauzu.

Erri eginarrazten nauzu.

Erri eginarazten didazu.

- Causé préfixé : 09/12 (dont 2 pour lesquels le préfixe peut être datif)

- Causé suffixé (datif) : 03/12

Dans le groupe intermédiaire la présence des formes à suffixe préfixé absolutif est en proportion plus forte; en effet, les locuteurs de parler traditionnel labourdin, où la préfixation peut être aussi bien absolutive que dative dans ces formes verbales sont au nombre de 2 seulement.

Le groupe des informateurs jeunes (-30 ans) scolarisés en immersion:

Irriarazten nauzu.

Irrinarazten nuzu.

Irriarazten nauzue.

Irri eginarazten didazu.

Zuk ni irriarazten nauzu.

Irriarazten nauzu.

Irrinaazten nuzu.
Irriarazten nauzu.

Irriarazten nauzu.

Irriarazten nauzu.

Irriarazten nauzu.

Irriarazten nauzu.

Irriarazten nauzu.

Irriarazten nauzu.

- Causé préfixé : 07 dont 1 ou 2 pour lesquels le préfixe peut être datif

- Causé suffixé (datif): 01 
Chez les jeunes ayant suivi une scolarité en basque le processus d'alignement absolutif déjà amorcé est pratiquement généralisé. Il est probable qu'en raison du caractère marqué de la construction, la règle du basque standard en la matière n'a pas été l'objet d'un enseignement scolaire spécifique. En conséquence, la tendance à la divergence perceptible dans les générations précédentes a suivi son cours, et la construction causative à objet absolutif s'est pratiquement généralisée.

Le groupe des informateurs jeunes (-30 ans) scolarisés hors immersion:

Irri egitenarazten (sic) nauzu.

Irri egiñarazten nauzu.

Irriarazten nauzue.

Irriarazten didazu.

Irri eginarazten nauzu.

Erriginazten deitazüt.

- Causé préfixé : 09

- Causé suffixé (datif): 02

\section{Irrinaazten nauzu.}

Zük erri eginazten naizü.

Irriarazten nauzu.

Irri inaazten naazu.

Erri eginazi naizie.

Chez les jeunes ayant été scolarisé uniquement en français, le phénomène observé dans l'autre groupe de jeunes est confirmé.

L'examen des réponses à travers les différents groupes fait nettement apparaître un phénomène de divergence, sans doute par influence du contact avec le français. Ce processus est en cours, car nous le voyons bien initié chez les informateurs les plus âgés et pratiquement généralisé chez les plus jeunes. La faible fréquence des occurrences de ce type de structure dans l'usage ordinaire de la langue fait que les prescriptions de la grammaire standard sont probablement sans grand effet. Il est d'ailleurs probable qu'elles ne sont pas l'objet d'un enseignement prescriptif spécifique dans le cadre scolaire. Par ailleurs, il est possible que ce phénomène soit encouragé dans les parlers ici étudiés par la tendance à traiter syntaxiquement les constructions à verbes léger du type $\mathrm{N}+$ egin comme des verbes totalement intransitifs où le nom incorporé a perdu certaine des propriétés syntaxiques des objets en basque (Oyharçabal 2006).

\subsubsection{L'emploi du réflexif lexical.}

Le basque réalise principalement ses formes réflexives par le recours à un syntagme réflexif léxicalisé comme un nom référentiel ordinaire (buru 'tête'), et prenant sa marque casuelle régulièrement, la personne grammaticale étant exprimée par un pronom personnel au génétif. Ainsi, je me suis suicidé se dit littéralement nire burua hil dut, lit. 'j'ai tué ma tête', et vous vous mentez, semblablement, zure buruari galdatu diozu, lit. 'vous dites des mensonges à votre tête'.

Il existe aussi un certain nombre de verbes qui expriment des procès du type se coiffer ou se laver, de nature réflexive, et que le basque exprime par l'emploi d'un verbe intransitif 
non actif. On peut alors parler d'expression du réfléxif par détransitivation, usage que l'on considère le plus souvent comme lexicalisé dans ces verbes.

La base de données inclut quelques questions regardant le réflexif comme notamment C32 : je ne m'aime pas beaucoup. Les résultats obtenus dans cette réponse font clairement apparaître un changement allant dans le sens d'un glissement vers l'emploi de la construction détransitivée pour exprimer le réflexif.

Informateurs âgés (+65 ans):

Eztut ne burua maite.

Nere burua hastio dut... erran nezake.

Eztut ne burua maite.

Ne burua ez dut maite.

Eztit ene büü izigarri maite.

Eztut ene burua maite.

Ene buria eztut maite.

Eztut nere burua maite biziki.
Ez dut ene burua biziki maite.

Eztizügü gue büia maite.

Ene büia eztüt hanitx maite.

Nere burua ez dut maite.

Ez dut ene burua anitz maite.

Eztut ene burua ainiz maite.

Eztut biziki maite nere burua.

- Réflexif lexical: $15 / 15$

- Détransitivation : 00/15

Ainsi qu'on peut le constater dans ce groupe tous les informateurs ont utilisé le réfléxif lexical avec le nom buru.

Le groupe des informateurs d'âge intermédiaire (40-60 ans) :

Ez dut maite ene burua.

Nere burua eztut biziki maite.

Eztit nihaun büia maite.

Eztit ene büia hanitx maite.

Ez dut ene buria biziki maite.

Niaun buria eztut biziki maite.
Eztut biziki maite ene burua.

Ez dut ene burua asko maite.

Ez naiz biziki gustatzen. ${ }^{3}$

Eztut ene burua biziki maite.

Ez naiz ainiz maite.

Ez dut ene burua maite biziki.

- Réflexif lexical : 10/12

- Détransitivation : 01/12

- Recours à un verbe psychologique du type 'piacere': 01/12

Dans le groupe intermédiaire un locuteur a utilisé le réflexif de détransitivation (ez naiz ainiz maite) avec le verbe maite izan d'usage normalement transitif. Un autre informateur, a un verbe du type 'piacere', à savoir gustatu sous un mode monoargumental pour restituer le réflexif (ez naiz biziki gustatzen). 
Le groupe des informateurs jeunes (-30 ans) scolarisés en immersion:

Nere burua eztut asko maite.

Eztut ene buria biziki maite.

Ez naiz ainitz maite.

Nere burua eztut asko maite.

Ez naiz ainitz maite.

Ez naiz ainitz maite.

Eztut ene buria ainitz maite.

Ez naiz asko maite.

Nere burua eztut ainitz maite.

- Réflexif lexical : 08/17

- Détransitivation : 09/17
Nere burua ez dut ainitz maite.

Ez dut ene burua sobera maite.

Eztut ene burua asko maite.

Ez naiz asko maite.

Ez naiz biziki maite.

Ez naiz ainitz maite.

Ez naiz asko maite.

Enaiz sobera maitatzen.

Dans le groupe des jeunes ayant suivi une scolarisation par immersion, les formes de réfléxif par détransitivation sont pratiquement à part égale avec celle à réflexif lexical, en nette rupture par rapport aux locuteurs des générations précédentes.

Le groupe des informateurs jeunes (-30 ans) scolarisés hors immersion:

Ene burua eztut ainitz maite.

Ez dut asko maite nire burua.

Ez dut biziki maite nere burua.

Ez niz asko maite.

Eztut asko maite nire burua.

Eztit ene büia maite hanitx.

Eztut nere burua aunitz maite.
Eztit ene büia maite hanitx.

Nere burua eztut añitz maite.

Niaun buria eztut aunitz maite.

Enüzü sobea maite.

Eztit nihauen büia izigarrin maite.

Ez naiz ainitz maite.

Enüzü hanitx maite.

- Réflexif lexical : 10/14

- Détransitivation : 04/14

Chez les jeunes locuteurs qui ne sont pas scolarisés dans le cadre de l'immersion (et, pour la plupart, ayant donc acquis le basque principalement par transmission familiale), la construction lexicale reste nettement majoritaire, bien que la détransitivation soit également présente de manière significative. Ce changement va à l'encontre des règles du basque standard, et représente une évolution en cours, probablement de façon plus marquée chez les jeunes locuteurs ayant acquis le basque principalement dans un environnement scolaire. Il est difficile de savoir si cette évolution représente une divergence par rapport aux dialectes d'outre-Bidassoa, car il est fort possible que dans ceux-ci aussi le même phénomène soit observable du fait d'un environnement également roman. 


\subsubsection{L'emploi du passé dans les complétives subjonctives.}

Le basque utilise des auxiliaires modaux pour exprimer le potentiel, l'impératif et le subjonctif. Dans ce dernier cas, il peut s'agir de phrases adjonctives exprimant la finalité, ou de phrases complétives sélectionnées typiquement par des prédicats de volonté ou ce crainte. En basque classique et standard, les phrases complétives subjonctives opérent obligatoirement un accord temporel avec le verbe matrice, de sorte que les formes complétives d'un verbe au passé sont elles aussi à une forme marquée, passée, ou éventuelle en souletin. Ceci est mis en évidence les exemples suivants:

$\begin{array}{llll}\text { Nahi } & \text { dut } & \text { gerta } & \text { dadin } \\ \text { vouloir.PRES } & \text { AUX } & \text { survenir } & \text { SUBJ.PRES }\end{array}$

'Je veux que cela arrive'.

$\begin{array}{llll}\text { Nahi } & \text { nuen } & \text { gerta } & \text { zedin } \\ \text { vouloir } & \text { PAS } & \text { survenir } & \text { SUBJ.PAS }\end{array}$

'Je voulais que cela arrivât'.

Concernant le subjonctif, les enseignants de basque ont fait deux observations: d'une part, une propention à y substituer des formes nominalisées; d'autre part, une tendance à la perte de la concordance temporelle dans leurs formes conjuguées. Le premier point correspond à une tendance ancienne et très répandue en basque, et nous ne l'examinerons pas ici. Le second regarde un élément qui rappelle une donnée observée en français, de sorte qu'il peut s'agir d'un effet du contact des langues. En effet, en français moderne, on observe une perte de la distinction temporelle au subjonctif, laquelle se traduit en particulier par la substitution du présent à l'imparfait du subjonctif (pour une analyse récente, voir Jeppensen Kragh 2010). Une question testait spécifiquement ce point dans le questionnaire NORANTZ : la question C181 : Je voulais que Beñat dise la vérité.

Avant de voir les réponses à cette question, il convient d'indiquer que le test n'est pas nécessairement opératoire pour chaque réponse, puisque le subjonctif peut être remplacé par une forme nominalisée, voire, plus rarement, par une protase d'éventuel dans le contexte de la phrase de test. Nous listerons ci-dessous toutes les réponses, mais nous ne prendrons en compte dans l'analyse que les réponses répondant au test (formes verbales en gras). ${ }^{25}$

25.- Comme on peut le voir les réponses non retenues pour le test sont celles où la complètive subjonctive n’a pas été utilisée, ou bien pour lesquelles l'informateur n'a pas utilisé une forme du passé avec le verbe matrice. 
Informateurs âgés (+65 ans):

Nai nuen Beñatek erran banindu egia.

Nahi nuen Beñatek egia erran zezan.

Nai nuen Beñatek egia erratia.

Nai nuke Beñatek egia erran baleza.

Nahi nikezü Beñatek egia erran dezan.

Nai nuen Beñatek erran dezan egia.

Nahi nuke Beñatek egia erran dezan.

Nahi nuen Beñatek egia erran zezan.

- Concordance passée: 06/08

- Pas de concordance:02/08
Nahi nuen Beñatek egia erran dezan.

Nahi nikezü Beñatek erran dezan egia.

Nahi nükin Beñatek erran lezan egia.

Naiko nuen Beñatek egia erran tzezan.

Nai nuke Beñatek erran dezan egia.

Nahi nian Beñatek erran lezan egia.

Nahi nuen Beñatek egia erran zezan.

Chez les informateurs les plus âgés, la concordance temporelle opère, bien que l'absence de concordance soit attestée.

Le groupe des informateurs d'âge intermédiaire (40-60 ans):

Nik nai nuen Beñatek erran dezan egia.

Beñatek egia erran dezan nai nuen.

Nahi nikezü Beñatek egia erran dezan.

Nahi nizün Beñatek egia erran dezan.

Nahi nuen Beñatek egia erran zezan.

Nahi nuen Beñatek egia erran tzezan.
Beñatek egia erran dezan nai nuen.

Nai nuen Beñatek egia erran zezan.

Nahi nuen Beñatek erran zezan egia.

Nahi nuen Beñatek egia erran zezan.

Nai nuke Beñatek egia erran dadin (sic). ${ }^{4}$

Nai nuen Beñatek egia erraitea.

- Concordance passée: 05/09

- Pas de concordance: 04/09

Chez les informateurs d'âge intermédiaire, la proportion de réponses respectant la concordance temporelle s'affaiblit, les deux usages étant à peu près au même niveau.

Le groupe des informateurs jeunes (-30 ans) scolarisés en immersion:

Nai nuen Beñatek egia erratea.

Nai nuen Beñatek egia erran tzezan.

Nai nuen Beñatek egia erran zezan.

Nai nuen Beñatek egia erran zezan.
Nai nuen Beñatek egia erran dezan.

Nai nuen Beñatek egia erran zezan.

Nai nuen Beñatek egia erran zezan.

Nai nuen Beñatek egia erran dezan. 
Nai nuen Beñatek egia erraitea.

Nai nuen Beñatek egia erraitea.

Nai nuen Beñatek egia erran zezan.

Nai nuen Beñatek egia erratea.

Nai nuen Beñatek egia erran zezan.

- Concordance passée: 07/10

- Pas de concordance : 03/10
Nai nuen Beñatek egia erraitea.

Nai nuen Beñatek egia erraitea.

Nai nuen Beñatek egia erran dezan.

Nai nuen Beñatek egia esatea (sic).

Chez les jeunes scolarisés par immersion, la concordance s'opère de manière prévalente. Pour ce profil d'informateur, l'emploi du subjonctif est probablement lié à un registre de langue soigné, pour lequel les prescriptions académiques ont un certain effet. Cependant, les cas de non-concordance demeurent présents.

Le groupe des informateurs jeunes (-30 ans) scolarisés hors immersion:

Nai nuen Beñatek egia erran dezan.

Nai nuen Beñatek egia erran dezan.

Nai nuen Beñatek egia erraitea.

Nai nuen Beñatek egia esan tzezan.

Nai nuen Beñatek egia erran dezan.

Nahi nizün Beñatek egia erran lezan.

Nai nuen Beñatek egia (...) erran dezan.
Nahi nizün Beñatek erran dezan egia.

Beñatek egia erran zezan nai nuen.

Beñatek egia erran tzezan nai nuen.

Nahi nizün Beñatek erran dezan egia.

Nahi nizun Beñatek egia lezan.

Nai nuen Beñatek egia erraitea.

Nahi nizün Beñatek egia erranik.

- Concordance passée: 05/11

- Pas de concordance : 06/11

Chez les jeunes scolarisés hors du système d'immersion, la tendance bien marquée chez les informateurs d'âge intermédiaire à ne pas respecter la concordance se retrouve dans des proportions semblables.

Le point étudié ici, fait clairement apparaître un changement en cours, dont la généralisation n'est pas cependant assurée. Il est possible que, chez les jeunes générations, la forme marquant la concordance tende à devenir une forme du registre soigné, puisque dans les conditions d'enquête, elle semble prévaloir chez les jeunes scolarisés en basque. Observons, que, dans le cas de ce changement, l'influence des dialectes d'outre-Bidassoa est probablement réduite, car ces dialectes n'utilisent que fort peu les formes conjuguées dans les complétives des verbes de volonté et assimilés. La question de la concordance ne concerne pas en effet les phrases nominalisées qui se substituent aux phrases subjonctives.

\subsubsection{L'emploi le l'inessif avec un verbe de mouvement.}

Le point suivant concerne un autre cas où un changement est susceptible de se produi- 
re. Il concerne une opposition dans le marquage locatif. En basque, un suffixe casuel différent est utilisé pour indiquer la localisation de but avec des verbes de déplacement (-ra joan 'aller à', -ra etorri 'venir à'), et celle concernant la localisation simple, sans déplacement (-an bizi izan 'vivre, habiter à', -an izan/egon 'être/se trouver à' ou bien associée à une pénétration (-an sartu 'entrer/pénétrer dans'). Dans le premier cas on emploie le suffixe -ra, adlatif, et dans le second cas, le suffixe -an, inessif. Cette distinction est transdialectale et présente en basque standard.

En français, ce marquage s'effectue par des prépositions, et la même préposition (à) peut être employée avec des verbes de déplacement (aller à, venir à, se déplacer à) pour indiquer la localisation du terme du déplacement, et pour indiquer une localisation avec des verbes sans déplacement (être à, habiter à, vivre à, etc.).

Les enseignants de basque ayant souvent à corriger les apprenants sur ce point, il est intéressant de voir ce qu'indique la base de données. Diverses questions portent sur le marquage adlatif où la localisation s'effectue par un objet inanimé (A 40, 41, 42, 43) et les réponses montrent chez quelques informateurs, très minoritaires, une certaine tendance à la perte du marquage adlatif. S'agissant d'une perte bien repérée dans les pratiques de prescription, on peut penser que dans les questions où l'adlatif est spécialement sollicité, les conduites d'autocorrection ont été favorisées, et que les usages moins contrôlés peuvent être différents. Pour vérifier ce point, on a choisi de présenter ici, les réponses à une autre question, où un autre point était ciblé, à savoir, l'emploi du partitif dans les phrases négatives (A19: Aucun enfant n'est venu à l'école aujourd'hui). Voici donc les réponses. La lecture des données concernant l'emploi de l'adlatif dans cette question doit être faite à la lumière de celle portant spécifiquement sur ce point. Les réponses où l'informateur n'a pas utilisé un verbe de déplacement sont rapportées, mais elles ne sont pas prises en compte dans l'analyse puisque le verbe n'y sélectionne pas l'adlatif (le syntagme locatif n'apparaît pas en gras dans les listes).

Informateurs âgés (+65 ans):

Ezta bate aurrik etorri eskolarat gaur. Haur biirik ezta etorri egun eskolarat. Gaur, aur biirik ezta eskolat etorri.

Aur batere ezta etorri egun eskolarat. Egün eztüzü haurrik bate e jin eskolalat. Egun haurrik ezta biirik jin eskolat. Egun haur bihiik ezta jin eskolarat. Haurrik ezta bat ere jin eguneskolarat.
Haur bihik ez da eskolara jin egun. Egün eztüzü haur bat e jin eskolalat. Haurrik ezta jin egün eskolalat. Aur biirik ez da etorri egun eskolarat. Haur bat ere ez da jin eskolarat egun. Ezta haurrik eskolalat jin egün. Haur bat ere eztuk jin eskolaat egun.

- Cas adlatif: $15 / 15$

- Cas inessif : 00/15

Chez les informateurs les plus âgés, tous ont répondu en utilisant un verbe de déplacement (etorri ou jin 'venir) et aucun n'a utilisé l'inessif à la place de l'adlatif sur le syntagme 
locatif sélectionné.

Le groupe des informateurs d'âge intermédiaire (40-60 ans):

Ezta aur bii bat ere etorri eskolarat egun.

Gaur eskolarat aurrik ezta etorri.

Eztüzü haur bakotxik e jin eskolalat egün.

Eztüzü bate haurrik jin egün eskolat.

Ezta haur bat ere jin eskolarat egun.

Haurrik ezta etorri gaur eskolara.
Aurrik ezta gaur etorri eskolara.

Gaur ezta aurrik agertu eskolan.

Ez da haurrik jin eskolarat egun.

Haur bihirik ezta izan eskolan eun.

Ez da aurrik izan eskolan.

Egun aurrik ezta etorri eskolarat.

- Cas adlatif: 09/09

- Cas inessif : 00/09

Chez les locuteurs d'âge intermédiaire trois réponses ont été données sans que le verbe de mouvement attendu soit employé. Ces réponses n'ont pas été comptabilisées, le verbe employé par les informateurs sélectionnant clairement l'inessif dans deux d'entre elles (izan 'être'), et pouvant sélectionner les deux marqueurs de localisation dans le cas du troisième (agertu 'apparaître'). Dans toutes les réponses où le verbe traduisant 'venir' a été utilisé, l'adlatif a été employé.

Le groupe des informateurs jeunes (-30 ans) scolarisés en immersion:

Ezta aurrik eskolara jin denik egun.

Ez da aurrik etorri gaur ikastolara.

Aurrik ezta etorri gaur eskolara.

Aurrak ez dira etorri ikastolara egun.

Ez da bat ere aurrik eskolara jin direnak gaur.

Ezta aur bat ere jin eskolara gaur.

Aurrak eztira etorri eskolara gaur.

Aurrik ezta eskolara etorri gaur.
Ezta aurrik etorri gaur eskolara.

Ezta aurrik jin eskolarat gaur.

Ezta aur bat ere eskolara etorri gaur.

Ezta aur bat ere etorri eskolarat gaur.

Aurrik ez da etorri ikastolara.

Aurretarik iñor ez da eskolara etorri.

Eztago aurrik eskolan etorri denik gaur.

- Cas adlatif: $14 / 15$

- Cas inessif : 01/15

Chez les jeunes scolarisés en immersion, un locuteur a utilisé l'inessif avec le verbe de déplacement. Il s'agit d'un locuteur ayant appris le basque dans le cadre scolaire et vivant dans un contexte urbain, où la présence sociale du basque est très faible.

Le groupe des informateurs jeunes (-30 ans) scolarisés hors immersion: 
Aurrik ez da eskolara etorri egun.

Ezta aur batere eskolara joan gaur.

Ezta aurrik etorri eskolarat gaur.

Ez da aurrik eskolara etorri denik gaur.

Ez da etorri aurrik eskolara gaur.

Egün haur bakotxik ezta jin eskolalat.

Ezta aur bat ere eskolarat etorri egun.
Haur batee eztüzü jun eskolala egün.

Aurrik ezta etorri gaur eskolara.

Haur bate ezta eskolara jin gaur.

Eztuzu bate haurrik eskolan egün.

Eztüzü haurrik jin eskolalat.

Aur bat ere ez da etorri eskolarat gaur.

Ez da haurrik jin eskolalat egun.

- Cas adlatif: $14 / 14$

- Cas inessif : 00/14

Dans le groupe des informateurs jeunes, scolarisés en dehors du système d'immersion, le seule réponse où l'adlatif n'a pas été employée est celle où le verbe de déplacement requérant ce cas n'apparaît pas (réponse non comptabilisée).

Ces résultats ne diffèrent pas radicalement de ceux recueillis dans les questions portant spécifiquement sur ce point (A40, 41, 42, 43). ${ }^{26}$ Comme on le voit, l'usage de l'adlatif en place de l'inessif avec les verbes de déplacement, bien qu'attesté, reste marginal dans la base de données NoRANTZ, même dans le cas où l'élicitation ne porte pas spécifiquement sur ce point.

\section{Conclusion}

Cet exposé nous a permis de présenter la base de données Norantz et quelques-uns les usages qu'elle peut offrir aux chercheurs et plus généralement aux professionnels de la langue et de son enseignement. Après avoir rappelé le contexte sociolinguistique du PBA, nous avons indiqué les conditions dans lesquelles ce projet a été réalisé et ses objectifs généraux. Visant à mettre en évidence les changements en cours dans la langue basque d'Aquitaine, l'enquête a été basée sur sa diversité des locuteurs, a la fois du point de vue de l'âge, et des lieux de vie, et, pour les plus jeunes, du mode de scolarisation concernant le basque. Pour les mêmes raisons, aucune condition relative à une maîtrise particulière de la langue basque n'a été recherchée chez les informateurs. Le fait d'être en mesure de répondre à ce long questionnaire, au cours d'entretiens non préparés ayant lieu en basque, représentant en lui-même la garantie d'une maîtrise minimale. Nous pensons que les conditions de recueil des données et, notamment, la variété des locuteurs, offre une bonne image de la grammaire utilisée par les locuteurs

26.- Parmi celles-ci, celle montrant l'écart le plus signficatif est A4l ('J'ai envoyé les enfants aux maisons derrière l'église'), pour laquelle 4 réponses ayant utilisé le verbe igorri 'envoyer' ont marqué la localisation avec l'inessif. Nous ne mentionnons pas ici, les cas où l'adlatif a été utilisé avec un verbe d'état (spécifiquement izan 'être'), et qui représentent le cas inverse de celui étudié dans le texte. Ces emplois sont également attestés dans la base de données, mais ils représentent un usage plus ancien dans le standard bas-navarrais-labourdin (Lafitte 1962). 
bascophones dans un usage non familier, tel que celui de l'enquête. Près d'une soixantaine de locuteurs ont répondu à 746 questions, dont 558 questions de morphologie fonctionnelle, au cours d'entretiens individuels non préparés, réalisés en trois sessions.

Il s’agit de la première étude générale, qui permet, dans le cadre du Pays Basque aquitain, de repérer, et d'organiser dans une base de données accessible en ligne, les usages linguistiques des divers types de locuteurs bascophones ordinaires, dans un ensemble considérable de points touchant principalement à la morphosyntaxe, et secondairement la phonétique. Toutes les données sont directement et aisément accessibles sous formes écrite et sonore. Elles dépassent les 44000 réponses.

Dans cette présentation, nous nous sommes attachés à mettre en évidence dans un premier temps des phénomènes de convergence en cours, avec la pénétration chez les jeunes générations de formes standards ou en provenance des dialectes les plus dynamiques d'outre-Bidassoa. Nous avons choisi pour cela les formes de suffixes casuels du datif pluriel et de l'adlatif approximatif, l'auxiliaire des verbes ditransitifs et, enfin, un marqueur de subordination, à savoir, le suffixe des complétives déclaratives en contexte négatif. Les données recueillies sont cohérentes et montrent clairement un effet de convergence sur ces divers éléments chez les jeunes générations. Les effets découlant, d'une part, de la standardisation, d'autre part, de l'influence grandissante des dialectes centraux d'outre-Bidassoa, très présents et dynamiques dans les instances créateurs de modèles linguistiques (littérature, enseignement, presse écrite ou audio-visuelle), sont d'ores et déjà nettement perceptibles dans le domaine morphosyntaxique, ce qui n'est pas le cas, signalons-le, dans le domaine phonétique (qui n’a pas évoqué dans cet article). Il convient de souligner cependant que, dans les cas de polymorphismes liés au registre, la méthode de d'enquête favorise les formes non familières des locuteurs ; on ne peut tirer d'enseignement quant à la persistance d'une éventuelle forme dialectale familière distincte, dans le registre correspondant.

Dans un second temps, quatre situations de divergence ont été examinées, probablement ou possiblement liées selon les cas à un effet du contact des langues et une influence du français. Le premier portait sur le marquage de l'argument causé des locutions verbales sémantiquement inergatives de type [NOM + egin 'faire'] causativées, où un marquage absolutif peut se substituer au marquage datif de la langue classique, usage que maintiennent les dialectes d'outre-Bidassoa et le basque standard. Il s'agit d'un phénomène relativement ancien, puisque déjà très présent dans le groupe des informateurs les plus âgés de la base de données Norantz. On a pu observer que le marquage absolutif (en divergence) se trouve renforcé chez les jeunes locuteurs, sans doute parce que s'agissant d'une structure complexe, d'une fréquence d'emploi relativement réduite, les démarches prescriptives associées en particulier à la scolarisation ont peu d'effets. Pour le second point, concernant la perte d'une concordance temporelle au passé dans les complétives subjonctives, il s'agit d'un phénomène de divergence que l'on voit progresser à travers les deux groupes d'âge les plus âgés, mais la tendance semble stoppée sinon inversée, chez les plus jeunes, en particulier ceux pour qui le basque est langue d'enseignement avec le français. Cependant, l'enquête ne permet pas de savoir si les formes de concordance sont également utilisées dans le registre familier. 
Les deux autres formes de changement évoquées dans l'article correspondent à des points que les enseignants et techniciens de la langue considèrent comme des lieux fréquents d'erreurs : l'un concerne la forme des emplois réfléxifs des verbes transitifs, l'autre, celui du marquage casuel des compléments locatifs des verbes de mouvement. Ici, les résultats de l'enquête ne viennent pas confirmer les impressions rapportées par les professionnels, car les formes divergentes, à savoir l'expression du réflexif par détransitivation, et l'usage de l'inessif en place de l'adlatif avec les verbes de mouvement, ne sont relevées dans la base de données que de façon marginale.

Sur le plan méthodologique, soulignons que l'élicitation par recours à la traduction de phrases élaborées en fonction des formes spécifiques objet d'enquête a donné de façon générale des résultats réellement satisfaisants. C'était au départ l'un des principaux éléments d'incertitude pour le groupe de recherche: serait-il possible que des locuteurs ordinaires, non préparés, parfois non alphabétisés en basque pour les deux groupes les plus âgés, répondent à un questionnaire basé sur la traduction, à la fois intensif et complexe, et même parfois réellement difficile? Et d'abord accepteraient-ils de s'astreindre devant un magnétophone à une telle tâche durant près de cinq heures (en trois séances séparées) ? L'expérience montre que des linguistes exercés en matière d'enquête de terrain sont en mesure de recueillir dans ce contexte des données de grande qualité en recourant à ce mode d'élicitation, même si les biais attachés à cette méthode ne sauraient être ignorés et doivent être pris en compte dans l'interprétation des résultats de l'enquête.

\section{Références}

Artiagoitia, Xabier. 2000. Hatsarreak eta Parametroak lantzen. Vitoria-Gasteiz: Arabako Foru Aldundia/UPV-EHU.

Aurrekoetxea, Gotzon. 1992. "Nafarroako euskara: azterketa dialektometrikoa", Uztaro 5, 59109.

Aurrekoetxea, Gotzon \& Videgain, X. 1993. "Euskal Herriko hizkuntz atlasa : galdesorta = cuestionario = questionnaire $:$ dialektologia / atlasgintza". Euskera 38, 527-647.

Aurrekoetxea, Gotzon, Videgain, X. \& Iglesias, A. (édit.). 2004. Bourciez bildumako euskal atlasa (BBEA): 1. lexikoa. ASJUren gehigarriak, XXXVIII-2, UPV/EHU, Bilbao.

Aurrekoetxea, Gotzon, Videgain, X. \& Iglesias, A. (édit.). 2005. Bourciez bildumako euskal atlasa (BBEA): 2. gramatika. ASJUren gehigarriak, XXXIX-1, UPV/EHU, Bilbao.

Baude, Olivier. (coord.). 2006. Corpus oraux, guide des bonnes pratiques. Paris et Orléans, Éditions du CNRS et Presses Universitaires d'Orléans.

Belleti, A. \& Rizzi, L. 1988. "Psych-Verbs and Theta-Theory", Natural Language and Linguistic Theory 6, 117-154.

Bonaparte, Louis Lucien. 1869. Le verbe basque en tableaux, London.

Camino, Iñaki. 1997. Aezkoako euskararen azterketa dialektologikoa. Iruñea: Nafarroako Gobernua. 
Camino, Iñaki. 2004. Hego-nafarrera. Iruñea: Nafarroako Gobernua.

Coyos, Jean-Baptiste. 1999. Le parler basque souletin des Arbailles: une approche de l'ergativité. Paris: L'Harmattan.

Epelde, Irantzu. 2004. Larresoroko euskara. Université du Pays Basque. Bilbao: UEU.

Epelde, Irantzu \& B. Oyharçabal \& J. Salaberria. 2011. "Norantz datu-basea: aurkezpena eta aditz komunztadurako datuak". ASJUren gehigarriak 69, 1-36.

Euskaltzaindia. 1999. Euskal Gramatika Lehen Urratsak-V (Mendeko perpausak-1). Bilbo: EHU \& Euskaltzaindia.

Euskaltzaindia. 2008. EHHA (Euskararen herri hizkeren atlasa) I et II, Euskaltzaindia-Académie de la Langue basque, Bilbao.

Euskaltzaindia. 2010. EHHA (Euskararen herri hizkeren atlasa) III, Euskaltzaindia-Académie de la Langue basque, Bilbao.

Euskaltzaindia. 2012. EHHA (Euskararen herri hizkeren atlasa) IV, Euskaltzaindia-Académie de la Langue basque, Bilbao.

Gómez, Ricardo. 2005. "De re etymologica: vasc.-(r)antz 'hacia”' in Etxebarria \& Knörr (édit.) Nerekin yaio nun: Txillardegiri omenaldia, IKER-17, Euskaltzaindia, Bilbao, 273-280.

Ibarra, Orreaga. 1995. Ultzamako hizkera: inguruko hizkerekiko harremanak. Iruñea: Nafarroako Gobernua.

Igartua, Iván. 2012. (édit.). Euskara eta inguruko hizkuntzak historian zehar. Vitoria-Gasteiz: Eusko Jaurlaritza.

Jeppensen Kragh, Kirsten. 2010. Le remplacement de l'imparfait du subjonctif par le présent du subjonctif considéré dans une perspective de grammaticalisation. Etudes romanes, 60, Museum Tuxculanum Press, Université de Copenhague, Copenhague.

Lafitte, Pierre. 1962 [1944]. Grammaire basque (navarro-labourdin littéraire). Baiona: Editions des Amis du Musée Basque et Ikas.

Lakarra, Joseba Andoni. 2006. "Protovasco, munda y otros: reconstrucción interna y tipología holística diacrónica”, Oihenart 21, 229-322.

Lakarra, Joseba Andoni. 2011. "Erro monosilabikoaren teoria eta aitzineuskararen berreraiketa: zenbait alderdi eta ondorio", Fontes Linguae Vasconum 113, 5-114.

Makazaga, Jesus Mari. 2010. Elgoibarko ahozko euskara. IKER-25, Euskaltzaindia, Bilbao.

Michelena, Luis. 1976. "La fragmentación dialectal: conocimientos y conjeturas". Lengua e historia. Madrid: Paraninfo, 73-85.

Michelena, Luis. 1988. Sobre historia de la lengua vasca. J. A. Lakarra (édit.), ASJUren gehigarriak 10, Donostia-San Sebastián.

Montoya, Estibalitz. 2004. Urdazubi eta Zugarramurdiko euskara. Iruñea: Nafarroako Gobernua.

Oyharçabal, Bernard. 1993. "Les documents recueillis lors des enquêtes linguistiques en Pays Basque durant la période révolutionnaire et le Premier Empire”, in J.B. Orpustan (édit.) La 
révolution française dans l'histoire et la littérature basques du XIXème siècle, éditions Izpegi, Baigorri, 63-119.

Oyharçabal, Bernard. 2006. «Basque Light Verb Constructions», in J. A. Lakarra \& J. I. Hualde (édit.), Studies in Basque and Historical Linguistics in Memory of R. L. Trask, ASJU, XL, 2006, 787-806.

Velasco, Ladislao de - . 1879. Los Euscaros en Alava, Guipúzcoa y Vizcaya. Sus orígenes, historia, lengua, leyes y tradiciones, Oliveres, Barcelone.

Yrizar, Pedro de. 1973. "Los dialectos y variedades de la lengua vasca. Estudio lingüístico-demográfico", BRSVAP XXIX, 3-78.

Zuazo, Koldo. 1998. "Euskalkiak, gaur”, Fontes Linguae Vasconum 78, 191-233.

Zuazo, Koldo. 2003. Euskalkiak: herriaren lekukoak. Donostia: Elkar. 